\title{
Alzheimer's disease brain contains tau fractions with differential prion-like activities
}

\author{
Longfei $\mathrm{Li}^{1,2+}$, Ruirui Shi ${ }^{1,2 \dagger}$, Jianlan Gu1,2, Yunn Chyn Tung ${ }^{1}$, Yan Zhou ${ }^{1,2}$, Dingwei Zhou ${ }^{2}$, Ruozhen Wu ${ }^{1,2}$,

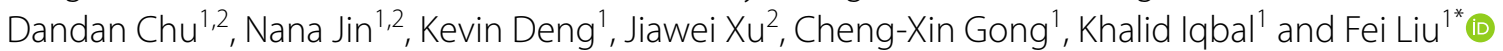

\begin{abstract}
Neurofibrillary tangles (NFTs) made of abnormally hyperphosphorylated tau are a hallmark of Alzheimer's disease (AD) and related tauopathies. Regional distribution of NFTs is associated with the progression of the disease and has been proposed to be a result of prion-like propagation of misfolded tau. Tau in AD brain is heterogenous and presents in various forms. In the present study, we prepared different tau fractions by sedimentation combined with sarkosyl solubility from AD brains and analyzed their biochemical and pathological properties. We found that tau in oligomeric fraction (O-tau), sarkosyl-insoluble fractions 1 and 2 ( $\mathrm{SI}_{1}$-tau and $\mathrm{SI}_{2}$-tau) and monomeric heat-stable fraction (HS-tau) showed differences in truncation, hyperphosphorylation, and resistance to proteinase $\mathrm{K}$. O-tau, $\mathrm{SI}_{1}$-tau, and $\mathrm{SI}_{2}$-tau, but not HS-tau, were hyperphosphorylated at multiple sites and contained SDS- and $\beta$-mercaptoethanol-resistant high molecular weight aggregates, which lacked the $\mathrm{N}$-terminal portion of tau. O-tau and $\mathrm{SI}_{2}$-tau displayed more truncation and less hyperphosphorylation than $\mathrm{SI}_{1}$-tau. Resistance to proteinase $\mathrm{K}$ was increased from $\mathrm{O}$-tau to $\mathrm{SI}_{1}$-tau to $\mathrm{SI}_{2}$-tau. O-tau and $\mathrm{SI}_{1}$-tau, but not $\mathrm{SI}_{2}$-tau or $\mathrm{HS}$-tau, captured tau from cell lysates and seeded tau aggregation in cultured cells. Heat treatment could not kill the prion-like activity of O-tau to capture normal tau. Hippocampal injection of O-tau into 18-month-old FVB mice induced significant tau aggregation in both ipsilateral and contralateral hippocampi, but $\mathrm{SI}_{1}$-tau only induced tau pathology in the ipsilateral hippocampus, and $\mathrm{SI}_{2}$-tau and $\mathrm{HS}$-tau failed to induce any detectable tau aggregation. These findings suggest that O-tau and $\mathrm{SI}_{1}$-tau have prion-like activities and may serve as seeds to recruit tau and template tau to aggregate, resulting in the propagation of tau pathology. Heterogeneity of tau pathology within AD brain results in different fractions with different biological and prion-like properties, which may pose a major challenge in targeting tau for development of effective therapeutic treatments.
\end{abstract}

Keywords: Alzheimer's disease, Tau pathology, Tau phosphorylation, Tau truncation, Prion-like seeding activity

\section{Introduction}

Alzheimer's disease (AD) is characterized pathologically by extracellular amyloid $\beta$ (A $\beta$ ) plaques and intracellular neurofibrillary tangles (NFTs) composed of abnormally hyperphosphorylated tau. Tau lesion (pretangles, neuropil threads, and NFTs), but not $A \beta$ plaque load, is

\footnotetext{
*Correspondence: fei.liu@opwdd.ny.gov

${ }^{\dagger}$ Longfei Li and Ruirui Shi contributed equally to this work

${ }^{1}$ Department of Neurochemistry, Inge Grundke-lqbal Research Floor,

New York State Institute for Basic Research in Developmental Disabilities,

1050 Forest Hill Road, Staten Island, NY 10314, USA

Full list of author information is available at the end of the article
}

correlated with cognitive disturbances $[2,5,23]$, suggesting a fundamental role of tau pathology in neurodegeneration of this disease.

In $\mathrm{AD}$ brain, tau pathology starts in the trans-entorhinal cortex, from where it spreads to limbic regions, followed by neocortical areas, according to the famous Braak stages $[7,8]$. The distribution of NFTs associates with the progression of this disease [8, 23]. After examining the brains of younger cohorts and discovering NFTs in the locus ceruleus of a subset of individuals, Braak revised that subcortical nuclei may actually be the site of the initial seed for tau propagation [12]. 
Recently, tau tracer retention measured by positron emission tomography also showed similar stages [35, $51,52]$. Thus, tau pathology in AD brain may spread along neuroanatomical connections, which underlies the progression of $\mathrm{AD}$.

The spatiotemporal spreading of tau pathology in $\mathrm{AD}$ was replicated recently in animal models. Clavaguera et al. injected brain extract from tau ${ }_{\mathrm{P} 301 \mathrm{~S}}$ transgenic mice into the brain of wild-type tau-expressing mice and induced tau aggregation not only at the injection sites, but also in the anatomically connected brain regions in a time-dependent manner [13], leading to introduction of the concept of "propagation of tau pathology." After this study, various mouse models were used to study the progressive propagation of tau pathology, including those using regional promoters, inoculation models, and viral models $[1,15,16,30,32$, $42,47]$. Tau pathology apparently radiates through the brain along synaptically connected pathways as the disease progresses.

As early as 1994, our group demonstrated that hyperphosphorylated cytosolic/oligomeric tau (AD p-tau) sequesters/captures normal tau in vitro to form filaments in a non-saturable manner [3], which was the first identification of prion-like activity of AD p-tau. Misfolded tau aggregates from brains of individuals with $\mathrm{AD}$ or tauopathies $[6,14,30,36]$ or from tau transgenic mouse brains [37] or generated in vitro [18, 27, 32, 50] are able to seed tau aggregation in cultured cells and in vivo. The seeding ability of tau from $\mathrm{AD}$ brains correlates positively with Braak stage and negatively with MMSE scores and precedes overt tau pathology [19]. In tau transgenic mice, tau seeds predict the spread of disease by appearing in brain regions prior to the appearance of any other pathological change [29]. Thus, the prion-like seeding activity of pathological tau may indicate the progression of tau pathology in AD.

Tau presents in different forms in AD brain [39], but its prion-like seeding activity is not well documented. By combining Kopke's and Guo's protocols [28, 39], we separated various tau fractions from AD brains, oligomeric fraction (O-tau), and sarkosyl-insoluble fractions 1 and $2\left(\mathrm{SI}_{1}\right.$-tau and $\mathrm{SI}_{2}$-tau), and heat-stable tau (HS-tau) (Fig. 1), by sedimentation in sarkosyl buffer and assessed their seeding activity by using new methods we recently developed [26]. We found that different AD tau fractions displayed different properties in truncation, hyperphosphorylation, resistance to proteinase $\mathrm{K}$, capturing normal tau in vitro and seeding tau aggregation in cultured cells and in vivo. O-tau and loose aggregates of tau in $\mathrm{SI}_{1}$-fraction showed prion-like activity, which is inert in compacted aggregates of tau in $\mathrm{SI}_{2}$-fraction and monomeric heat-stable tau.

\section{Materials and methods}

\section{Isolation of various tau fractions from AD brain}

Frozen brain tissue samples from autopsied and histopathologically confirmed AD cases with Braak Stages V and VI were obtained from the Brain Tissue Resource Center, McLean Hospital, Belmont, MA, USA. The use of autopsied frozen human brain tissue was in accordance with the National Institutes of Health guidelines and was exempted by the Institutional Review Board (IRB) of the New York State Institute for Basic Research in Developmental Disabilities because "the research does not involve intervention or interaction with the individuals" nor "is the information individually identifiable".

Tau fractions were isolated from autopsied and frozen AD cerebral cortex by a combination of the protocols described by Kopke and by Guo [28, 39]. Briefly (Fig. 1), $10 \%$ brain homogenate prepared in homogenization buffer (20 mM Tris-HCl, pH 8.0, $0.32 \mathrm{M}$ sucrose, $10 \mathrm{mM}$ $\beta$-mercaptoethanol ( $\beta$-ME), $5 \mathrm{mM} \mathrm{MgSO}_{4}, 1 \mathrm{mM}$ EDTA, $10 \mathrm{mM}$ glycerophosphate, $1 \mathrm{mM} \mathrm{Na} \mathrm{VO}_{4}, 50 \mathrm{mM} \mathrm{NaF}$, $2 \mathrm{mM}$ benzamidine, $1 \mathrm{mM}$ 4-(2-aminoethyl) benzenesulfonyl fluoride hydrochloride (AEBSF), and $10 \mu \mathrm{g} / \mathrm{ml}$ each of aprotinin, leupeptin, and pepstatin) was centrifuged at $27,000 \times g$ for $30 \mathrm{~min}$. The pellet was saved for sarkosylinsoluble tau (SI-tau) preparation. The supernatant was further centrifuged at $235,000 \times g$ for $30 \mathrm{~min}$, and the resulting pellet, i.e., oligomeric tau-enriched fraction (O-tau), was collected and washed twice with saline and then resuspended in saline (Fig. 1). The supernatant, Suptau (Fig. 1), was used for HS-tau preparation.

Sarkosyl-insoluble aggregated tau preparation: The pellet from the $27,000 \times g$ centrifugation above was homogenized in the homogenization buffer containing $0.1 \%$ sarkosyl and centrifuged at $10,000 \times g$ for $10 \mathrm{~min}$. The supernatant was adjusted to $1 \%$ sarkosyl, incubated for $1 \mathrm{~h}$ at room temperature (RT), and centrifuged at $235,000 \times g$ for $45 \mathrm{~min}$. The pellet was washed once with $1 \%$ sarkosyl-homogenization buffer and washed twice with saline to obtain $\mathrm{SI}_{1}$-tau (Fig. 1). The pellet from the $10,000 \times g$ centrifugation above was incubated with $1 \%$ Triton X-100 in homogenization buffer for $30 \mathrm{~min}$ at RT and centrifuged for $1 \mathrm{~h}$ at $100,000 \times g$. The resulting pellet was incubated in 1\% sarkosyl in homogenization buffer for $1 \mathrm{~h}$ at RT and centrifuged at $100,000 \times g$ for $45 \mathrm{~min}$. The resulting pellet was washed once with $1 \%$ sarkosyl in homogenization buffer and twice with saline and collected as $\mathrm{SI}_{2}$-tau (Fig. 1).

HS-tau preparation: The supernatant from the $235,000 \times g$ centrifugation above was adjusted to $0.75 \mathrm{M}$ $\mathrm{NaCl}$ and $10 \mathrm{mM} \beta$-ME, heated for $5 \mathrm{~min}$ at $100{ }^{\circ} \mathrm{C}$, and centrifuged at $235,000 \times g$ for $45 \mathrm{~min}$. The resulting supernatant was dialyzed against saline; the tau in this pool was termed HS-tau (Fig. 1). 


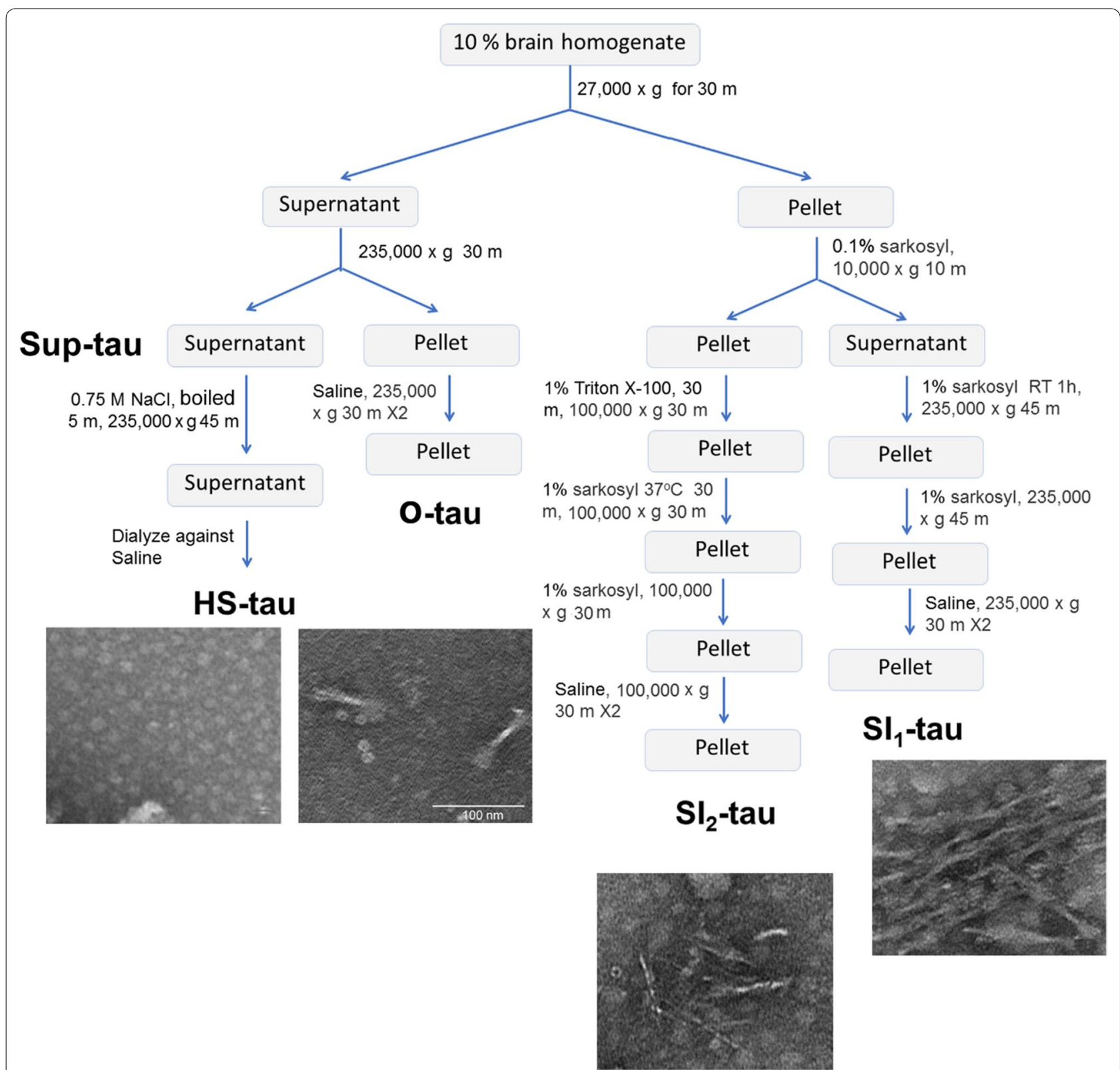

Fig. 1 The main steps used for isolation of various tau fractions from AD brains and representative images of each fraction obtained by negative staining electron microscopy

The tau preparations derived from $\mathrm{AD}$ brain described above were probe-sonicated for $5 \mathrm{~min}$ at $20 \%$ power and stored at $-80^{\circ} \mathrm{C}$ until use.

\section{Negative staining electron microscopy}

Various tau fractions were placed on 300 meshed carboncoated copper grids for $1 \mathrm{~min}$, stained with one drop of $2 \%$ Phosphotungstic acid for $1 \mathrm{~min}$, and visualized with Hitachi HT7700 transmission electron microscope.

\section{Cell culture and transfection}

HEK-293FT cells and HeLa cells were maintained in Dulbecco's modified Eagle's medium (DMEM) supplemented with $10 \%$ fetal bovine serum (FBS) (ThermoFisher Scientific, Waltham, MA, USA) at $37{ }^{\circ} \mathrm{C}(5 \%$ $\mathrm{CO}_{2}$ ). Transfections were performed with FuGENE HD (Promega, Madison, WI, USA) according to the manufacturer's instructions. 


\section{Western blots and immuno-dot blots}

Samples were denatured by boiling in Laemmli buffer for $5 \mathrm{~min}$. Protein concentration was measured using the Pierce ${ }^{\mathrm{TM}} 660 \mathrm{~nm}$ Protein Assay Kit (ThermoFisher Scientific). Samples were subjected to SDS-PAGE and transferred onto polyvinylidene fluoride membrane (Millipore Sigma, Burlington, MA, USA). The membrane was subsequently blocked with $5 \%$ fat-free milk-TBS (Tris-buffered saline) for $30 \mathrm{~min}$, incubated with primary antibodies (Table 1) in 5\% fat-free milk-TBS overnight, washed with TBST (TBS containing 0.05\% Tween 20), incubated with peroxidase (HRP)-conjugated secondary antibodies (Jackson ImmunoResearch Laboratories, West Grove, PA, USA), washed with TBST, incubated with the ECL Western Blotting Substrate (ThermoFisher Scientific) and exposed to HyBlot $\mathrm{CL}^{\circledR}$ autoradiography film (Denville Scientific Inc., Holliston, MA, USA). Specific immunosignal was quantified by using the Multi Gauge software V3.0 from Fuji Film (Minato, Tokyo, Japan).

Tau level in samples was assayed by immuno-dot blots as described previously. Briefly, various amounts of a sample were applied onto nitrocellulose (NC) membrane (Schleicher and Schuell, Keene, NH, USA) at $5 \mu$ l per grid of $7 \times 7 \mathrm{~mm}$ in size. The blot was placed in a $37^{\circ} \mathrm{C}$ oven for $1 \mathrm{~h}$ to allow the protein to bind to the membrane. Then, the membrane was processed as for Western blots described above by using a mixture of R134d and 92e pan tau antibodies as primary antibodies.

\section{Proteolysis of $A D$ tau fractions by proteinase $K$}

AD tau fractions $(2.5 \mathrm{mg} / \mathrm{ml})$ were incubated with various concentration of proteinase $\mathrm{K}$ in $10 \mathrm{mM}$ Tris $-\mathrm{HCl}$, $\mathrm{pH}$ 7.4, for $10 \mathrm{~min}$ at RT. The reaction was stopped by boiling in Laemmli buffer for 5 min. Proteolyzed tau was analyzed by Western blots.

\section{Tau capture/sequestration assay}

$\mathrm{Tau}_{151-391}$ tagged with hemagglutinin (HA) was overexpressed in HEK-293FT cells. The cells, $48 \mathrm{~h}$ after transfection, were lysed in phosphate-buffered saline (PBS) containing $50 \mathrm{mM} \mathrm{NaF}, 1 \mathrm{mM} \mathrm{Na} \mathrm{VO}_{4}, 1 \mathrm{mM}$ AEBSF, $5 \mathrm{mM}$ benzamidine, and $10 \mu \mathrm{g} / \mathrm{ml}$ each of aprotinin, leupeptin, and pepstatin by probe sonication at $20 \%$ power for $2 \mathrm{~min}$. The cell lysates were centrifuged for $10 \mathrm{~min}$ at $10,000 \times g$. The extract containing HA-tau ${ }_{151-391}$ was stored at $-80^{\circ} \mathrm{C}$ until use.

Table 1 Antibodies used in this study

\begin{tabular}{|c|c|c|c|c|}
\hline Antibody & Type & Specificity & Species & Source/reference (cat/lot) \\
\hline $43 \mathrm{D}$ & Mono- & Pan-tau (a.a. 6-18) & M & In house/Biolegend (816601) \\
\hline $92 \mathrm{e}$ & Poly- & Pan-tau & $\mathrm{R}$ & In house $[40]$ \\
\hline R134d & Poly- & Pan-tau & $\mathrm{R}$ & In house [40] \\
\hline $111 e$ & Poly- & Pan-tau & $\mathrm{R}$ & In house [40] \\
\hline $113 e$ & Poly- & Tau (a.a. 19-32) & $\mathrm{R}$ & In house $[40]$ \\
\hline HT7 & Mono- & tau (a.a. 159-163) & M & ThermoFisher (MN10000/LK152163) \\
\hline Tau5 & Mono- & tau (a.a. 210-230) & M & Millipore (MAB361/1816394) \\
\hline 77G7 & Mono- & tau (a.a. 244-368) & M & In house/Biolegend (816701) \\
\hline RD3 & Mono- & 3R-tau & M & Millipore (05-803/JBC1863429) \\
\hline RD4 & Mono- & 4R-tau & M & Millipore (05-804/2073108) \\
\hline QCB23070 & Poly- & Up-tau (S46) & $\mathrm{R}$ & Gong et al. [21] \\
\hline Tau-1 & Mono- & Up-tau (S199/202) & M & Dr. Lester I. Binder \\
\hline AT8 & Mono- & p-tau (S202/T205) & M & ThermoScientific (MN1020/PI205175) \\
\hline Anti-pT205 & Poly- & p-tau (T205) & $\mathrm{R}$ & Invitrogen (44738G/RD214239) \\
\hline Anti-pS214 & Poly & p-tau (S214) & $\mathrm{R}$ & Invitrogen (44742G/0500B) \\
\hline Anti-pT217 & Poly- & p-tau (T217) & $\mathrm{R}$ & Invitrogen (44744/785771A) \\
\hline AT180 & Mono- & p-tau (T231) & M & Invitrogen (MN1040/SH2406086) \\
\hline Anti-pS262 & Poly- & p-tau (S262) & $\mathrm{R}$ & Invitrogen (44-750G/0204) \\
\hline PHF1 & Mono- & p-tau (S396/404) & M & Dr. Peter Davies \\
\hline $\mathrm{R} 145$ & Poly- & p-tau (S422) & $\mathrm{R}$ & In house $[40]$ \\
\hline Anti-GAPDH & Poly- & GAPDH & $\mathrm{R}$ & Sigma (G9545/015M4824V) \\
\hline Anti-HA & Mono- & $\mathrm{HA}$ & M & Sigma (H9658/112M4841) \\
\hline Anti-HA & Poly- & $\mathrm{HA}$ & $\mathrm{R}$ & Sigma (H6908/115M4872V) \\
\hline
\end{tabular}

Mono- monoclonal, $p$ - phosphorylated, up- unphosphorylated, Poly- polyclonal, $M$ mouse, $R$ rabbit 
Various amounts of $\mathrm{AD}$ tau fractions were dotted on nitrocellulose membrane and dried at $37^{\circ} \mathrm{C}$ for $1 \mathrm{~h}$. The membrane was blocked with 5\% fat-free milk-TBS for $1 \mathrm{~h}$ and incubated with the above cell extract containing HAtau $_{151-391}$ overnight. After washing with TBST, captured HA-tau ${ }_{151-391}$ was detected by incubating with anti-HA in 5\% milk-TBST and processed as described above for immuno-dot blots.

\section{AD tau fractions seed tau aggregation in cultured cells} HEK-293FT cells were transfected with pCI/HA$\operatorname{tau}_{151-391}$ with FuGENE HD. Similar levels of tau in various AD tau fractions were mixed with Lipofectamine 2000 (3\% in Opti-MEM) (ThermoFisher Scientific) in $50 \mu \mathrm{l}$ for $20 \mathrm{~min}$ at RT and added to the cell cultures in 24-well plate after $6 \mathrm{~h}$ transfection. The cells were then lysed in RIPA buffer (50 mM Tris- $\mathrm{HCl}, \mathrm{pH} 7.4,150 \mathrm{mM}$ $\mathrm{NaCl}, 1 \% \mathrm{NP}-40,0.5 \%$ sodium deoxycholate, and $0.1 \%$ SDS) containing $50 \mathrm{mM} \mathrm{NaF}, 1 \mathrm{mM} \mathrm{Na} \mathrm{VO}_{4}, 1 \mathrm{mM}$ AEBSF, $5 \mathrm{mM}$ benzamidine, and $10 \mu \mathrm{g} / \mathrm{ml}$ each of aprotinin, leupeptin, and pepstatin for $20 \mathrm{~min}$ on ice after $42 \mathrm{~h}$ treatment. The cell lysates were centrifuged at $130,000 \times g$ for $45 \mathrm{~min}$, and the resulting pellet was washed with RIPA buffer. The supernatants were pooled together as RIPAsoluble fraction and the pellet contained RIPA-insoluble fraction. Levels of RIPA-insoluble and -soluble tau were analyzed by Western blots developed with anti-HA.

To visualize tau aggregates induced by various AD tau fractions in cells, HA-tau ${ }_{151-391}$ was overexpressed in HeLa cells and treated with tau fractions for $42 \mathrm{~h}$, as described above. The cells were then fixed for $15 \mathrm{~min}$ with $4 \%$ paraformaldehyde in phosphate buffer, washed with PBS, and treated with $0.3 \%$ Triton in PBS for $15 \mathrm{~min}$ at RT. After blocking with $5 \%$ newborn goat serum, $0.1 \%$ Triton X-100, and 0.05\% Tween 20 in PBS for $30 \mathrm{~min}$, the cells were incubated with anti-HA in blocking solution overnight at $4{ }^{\circ} \mathrm{C}$, washed with PBS, and incubated with Alexa Fluor 488-conjugated-secondary antibody for $2 \mathrm{~h}$ at RT. After washing with PBS, the cells were mounted with ProLong ${ }^{\mathrm{TM}}$ Gold antifade reagent (ThermoFisher Scientific) and observed with a Nikon confocal microscope. The experiment was performed in triplicate wells and eight fields were photographed from each well of each group. Percentage of cells with aggregated tau was determined. Each experiment was repeated at least twice.

\section{Tau pathology templated by $A D$ tau fractions in vivo}

Similar amounts of tau in O-tau, $\mathrm{SI}_{1}$-tau, $\mathrm{SI}_{2}$-tau, and HS-tau determined by immuno-dot blots with mixture of two polyclonal pan-tau antibodies (92e and R134d) were injected into the hippocampus unilaterally in 18-monthold male and female FVB mice. Mice were deeply anesthetized and transcardially perfused with saline followed by buffered $4 \%$ paraformaldehyde 3 months after tau injection. Brain was post-fixed in the same fixation buffer overnight at $4{ }^{\circ} \mathrm{C}$ and dehydrated in buffered $30 \%$ sucrose solution. Brain was then cut into $40-\mu \mathrm{m}$ serial coronal sections by using a freezing microtome, and the sections were collected in a 12-well plate containing antifreeze solution (30\% glycerol and 30\% ethylene glycol in 40\% PBS) in sequence, and the free-floating sections were preserved in antifreeze solution at $-20{ }^{\circ} \mathrm{C}$ before immunohistochemical staining.

\section{Immunofluorescent staining}

Brain sections from one well of a 12-well plate per mouse were washed with PBS and treated with $0.3 \%$ Triton in PBS for $15 \mathrm{~min}$ at RT. After blocking with 5\% newborn goat serum, $0.1 \%$ Triton $\mathrm{X}-100$, and $0.05 \%$ Tween 20 in PBS for $30 \mathrm{~min}$, the sections were incubated with AT8 antibody in blocking solution overnight at $4{ }^{\circ} \mathrm{C}$, washed with PBS, and incubated with Alexa 555-conjugatedsecond antibody for $2 \mathrm{~h}$. Hoechst dye was used to stain nuclei. After washing with PBS, sections were mounted on microscope slides, air-dried, mounted with ProLong $^{\mathrm{TM}}$ Gold antifade reagent (ThermoFisher Scientific), and set under a coverslip before imaging using a Leica TCS SP5 confocal microscope. For quantification, average AT8-positive neurons in contralateral and ipsilateral hippocampi were counted from three brain sections per mouse.

\section{Statistical analysis}

The GraphPad Prism 6 software was used for statistical analysis. Results were analyzed by one-way ANOVA followed with Tukey's multiple comparisons test or by twoway ANOVA followed by Sidak's multiple comparisons test for multiple-group analysis.

\section{Results AD tau fractions are truncated differentially}

Tau in AD brain appears in various pools and in monomeric, oligomeric, and filamentous forms [39]. In addition to aggregated tau, AD brain also expresses similar levels of normal tau [38], which is heat-stable [17]. Aggregated tau is sarkosyl-insoluble [22]. Normal tau and pathological tau can be separated by sedimentation. In the present study, by combining Kopke's and Guo's protocols [28, 39], we isolated various tau fractions-O-tau, $\mathrm{SI}_{1}$-tau, $\mathrm{SI}_{2}$-tau, and $\mathrm{HS}$ tau-from AD brains (Fig. 1). Negative staining microscopy showed paired helical filaments in fraction $\mathrm{SI}_{1}$-tau, mostly straight filaments in $\mathrm{SI}_{2}$-tau, short filaments in O-tau, and non-filament in HS-tau (Fig. 1).

It is widely believed that truncation of tau plays a critical role in tau pathogenesis $[49,54,58]$. To reveal 
the truncation of various $\mathrm{AD}$ tau fractions, we isolated aggregated O-tau, $\mathrm{SI}_{1}$-tau, and $\mathrm{SI}_{2}$-tau from four $\mathrm{AD}$ cerebral cortices and analyzed tau protein patterns by using polyclonal and monoclonal pan-tau antibodies to various regions of the protein (Fig. 2a). HS-tau from one AD brain was used as a reference. In general, we found SDSand $\beta$-ME-resistant high-molecular weight-tau (HMWtau) in O-tau, $\mathrm{SI}_{1}$-tau, and $\mathrm{SI}_{2}$-tau detected by $\mathrm{R} 134 \mathrm{~d}, 92 \mathrm{e}$, and $111 \mathrm{e}$ (Fig. 2b). Heat treatment is known to remove aggregated tau [48]. We found no detectable HMW-tau in HS fraction (Fig. 2b). Compared with blots developed with antibodies R134d and 92e, a lesser amount of tau in $\mathrm{SI}_{2}$ fraction was detected with antibody $111 \mathrm{e}$ (Fig. 2b).

To determine tau truncations, O-tau, $\mathrm{SI}_{1}$-tau, $\mathrm{SI}_{2}$-tau, and HS-tau were analyzed by Western blots developed with a battery of tau antibodies targeting specific

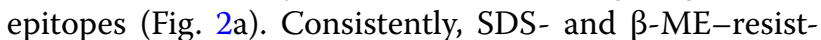
ant $\mathrm{HMW}$-tau was seen in $\mathrm{O}$-tau, $\mathrm{SI}_{1}$-tau, and $\mathrm{SI}_{2}$-tau but not in HS-tau (Fig. 2d). HMW-tau was detected by Tau5, 77G7, RD3, RD4, and Tau46, but was not, or only weakly, stained by N-terminal antibodies 43D, 113e, and HT7 (Fig. 2d), suggesting that it is truncated at the N-terminus. Immunoactivities of the $\mathrm{N}$-terminal antibodies 43D, 113e, and $\mathrm{HT} 7$ were less in $\mathrm{O}$-tau and $\mathrm{SI}_{2}$-tau than in $\mathrm{SI}_{1}$-tau (Fig. 2d, e). Tau5 immunoactivity was slightly higher in $\mathrm{O}$-tau and $\mathrm{SI}_{1}$-tau than in $\mathrm{SI}_{2}$-tau (Fig. 2d, e). Interestingly, immunoactivities of three antibodies against the microtubule-binding repeats, 77G7, RD3 and RD4, were similarly decreased from $\mathrm{O}$-tau, $\mathrm{SI}_{1}$-tau to $\mathrm{SI}_{2}$-tau (Fig. 2d, e), but $77 \mathrm{G} 7$ revealed strong immunoactivity toward O-tau, $\mathrm{SI}_{1}$-tau, and $\mathrm{SI}_{2}$-tau (Fig. $2 \mathrm{~d}$, e). Compared with $\mathrm{SI}_{1}$-tau, a lower level of tau in $\mathrm{O}$ - and $\mathrm{SI}_{2}$-fractions was detected by Tau46 (Fig. 2d, e). Taken together, these results suggest that $\mathrm{AD}$ tau fractions are truncated differentially. $\mathrm{O}$-tau and $\mathrm{SI}_{2}$-tau are truncated more at both $\mathrm{N}$ - and C-termini, compared with $\mathrm{SI}_{1}$-tau.

\section{AD tau fractions are hyperphosphorylated differentially}

Tau is hyperphosphorylated and aggregated into NFTs in AD and related tauopathies [24, 25, 33, 41]. To determine tau hyperphosphorylation, four AD tau fractions were subjected to Western blots developed with sitespecific and phosphorylation-dependent tau antibodies. Consistently, we found HMW-tau in O-tau, $\mathrm{SI}_{1}$-tau, and $\mathrm{SI}_{2}$-tau, but not in HS-tau. HMW-tau was detected by all phospho-tau antibodies (Fig. 3a). O-tau, $\mathrm{SI}_{1}$-tau, and
$\mathrm{SI}_{2}$-tau were hyperphosphorylated at Thr205, Thr212, Thr217, Ser231, Ser262, Ser396/404, and Ser422, but HS-tau was not, or only a little, phosphorylated at these sites (Fig. 3a, b). However, HS-tau was phosphorylated at Ser199 and Ser214 (Fig. 3a, b). Thus, O-, $\mathrm{SI}_{1^{-}}$, and $\mathrm{SI}_{2}$-tau fractions, but not HS-tau, are hyperphosphorylated at multiple sites. The SDS- and $\beta$-ME-resistant HMW-tau is hyperphosphorylated.

$\mathrm{O}$-tau and $\mathrm{SI}_{2}$-tau showed similar phosphorylation levels, but both were less phosphorylated than $\mathrm{SI}_{1}$-tau at Ser199, Thr205, Thr212, Ser214, Thr217, and Ser422 (Fig. 3a, b). O-tau and $\mathrm{SI}_{1}$-tau were phosphorylated similarly, but $\mathrm{SI}_{2}$-tau was less phosphorylated at Ser231 and Ser262, the sites in, or close to, microtubule-binding repeats (Fig. 3a, b). Similar phosphorylation levels of tau were observed in three AD tau fractions at Ser396/404 (Fig. 3a, b). Thus, these results suggest that O-tau, $\mathrm{SI}_{1}$-tau, and $\mathrm{SI}_{2}$-tau are hyperphosphorylated differentially. $\mathrm{SI}_{1}$-tau may be more hyperphosphorylated than $\mathrm{O}$-tau and $\mathrm{SI}_{2}$-tau at most phosphorylation sites.

We also analyzed un-phosphorylated tau in these AD tau fractions by Western blots developed with antibodies to un-phosphorylated tau: Ser46 (Up-Ser46) and Tau-1 (Up-Ser195-202). We found no or very little un-phosphorylated tau at Ser46 and at Ser195-202 (Tau-1) in O-tau, $\mathrm{SI}_{1}$-tau, and $\mathrm{SI}_{2}$-tau, but marked levels in HS-tau (Fig. 3c, d). Moreover, $\mathrm{SI}_{1}$-tau contained more un-phosphorylated tau than $\mathrm{O}$-tau and $\mathrm{SI}_{2}$-tau (Fig. 3c, d). These results showed that $\mathrm{O}$-tau, $\mathrm{SI}_{1}$-tau, and $\mathrm{SI}_{2}$-tau are hyperphosphorylated, and HS-tau is less phosphorylated, at Ser46 and tau-1 sites. In addition to hyperphosphorylated tau, a small fraction of $\mathrm{SI}_{1}$-tau also is un-phosphorylated at Ser46 and tau- 1 sites.

\section{$A D$ tau fractions are resistant to proteinase $K$ differentially}

It is well known that tau in NFT is resistant to proteinase $K$ [56]. To determine the sensitivity of these AD tau fractions to proteinase $\mathrm{K}$, we incubated them with various concentrations of proteinase $K$ for $10 \mathrm{~min}$ at room temperature and analyzed the digestion products by Western blots developed with 77G7 and with a mixture of R134d and 92e. We found that proteinase $\mathrm{K}$ proteolyzed O-tau, $\mathrm{SI}_{1}$-tau, and $\mathrm{SI}_{2}$-tau to small-molecular weight products that immunoreacted with $77 \mathrm{G} 7$ (Fig. 4c), but weakly with R134d/92e (Fig. 4a) in a dosedependent manner. The greater reductions of tau by

(See figure on next page.)

Fig. 2 AD tau fractions are truncated at different sites. a. Schematic showing the position of epitopes of various tau antibodies used to analyze tau truncation. b-e Western blots of O-tau, $\mathrm{SI}_{1}$-tau, $\mathrm{SI}_{2}$-tau, and HS-tau from AD brains developed with polyclonal pan-tau antibodies (R134d, 111e, and 92e) (b), or with epitope-specific antibodies (d). The levels of tau in the four pools determined by the individual antibody were quantified densitometrically and are presented as mean $\pm \mathrm{SD}(\mathbf{c}, \mathbf{e}) .{ }^{*} p<0.05 ;{ }^{* *} p<0.01$. A.U., arbitrary units 


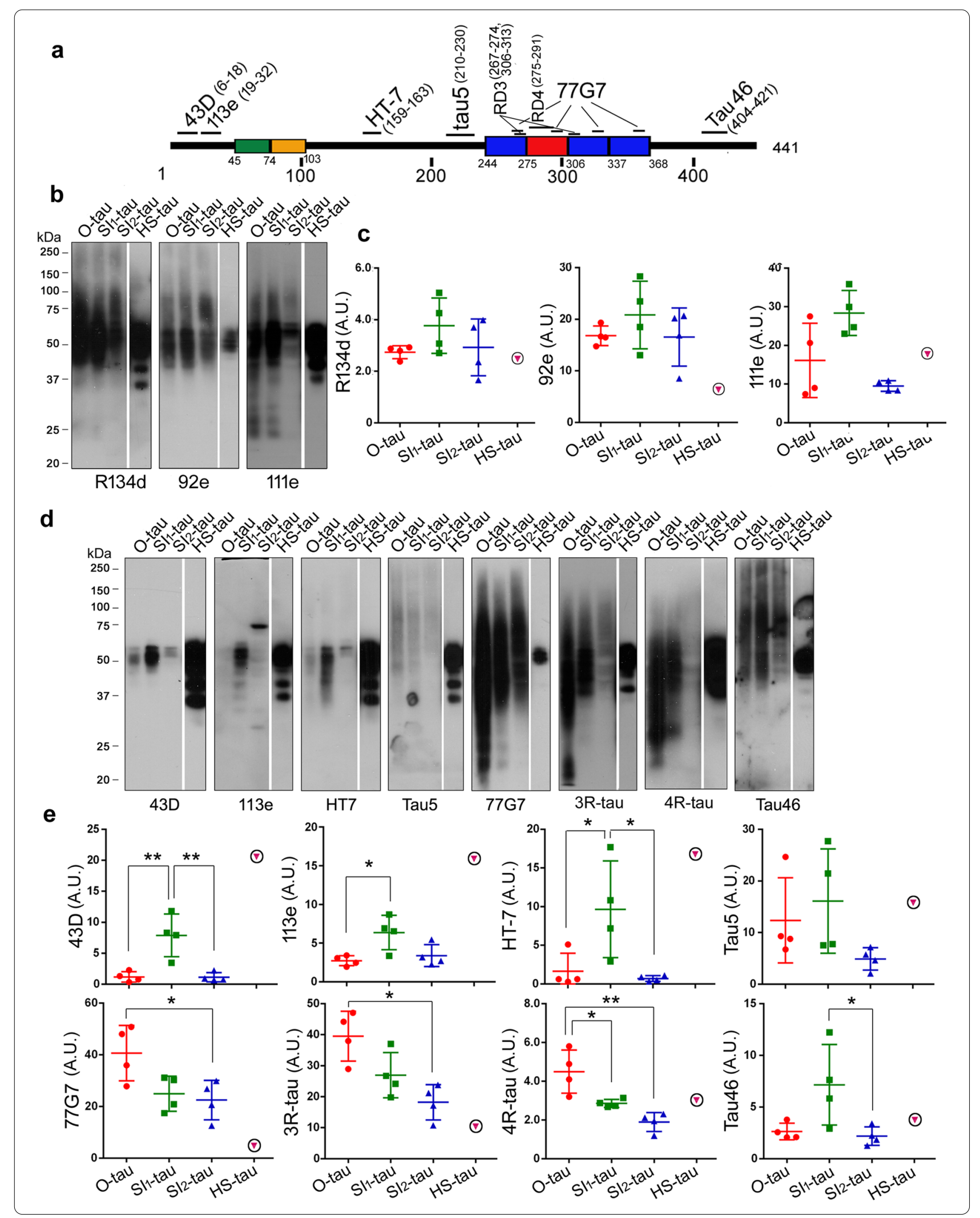




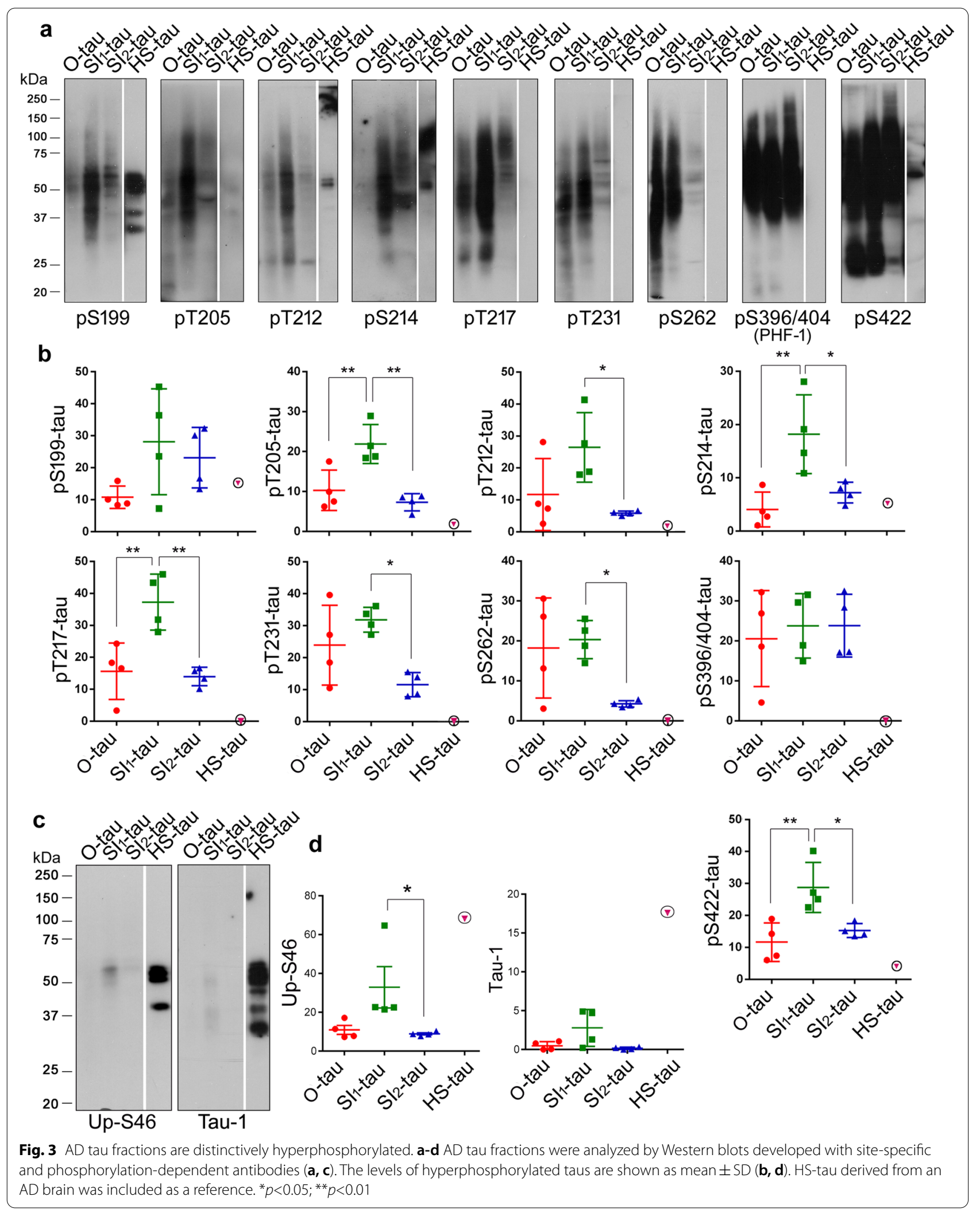



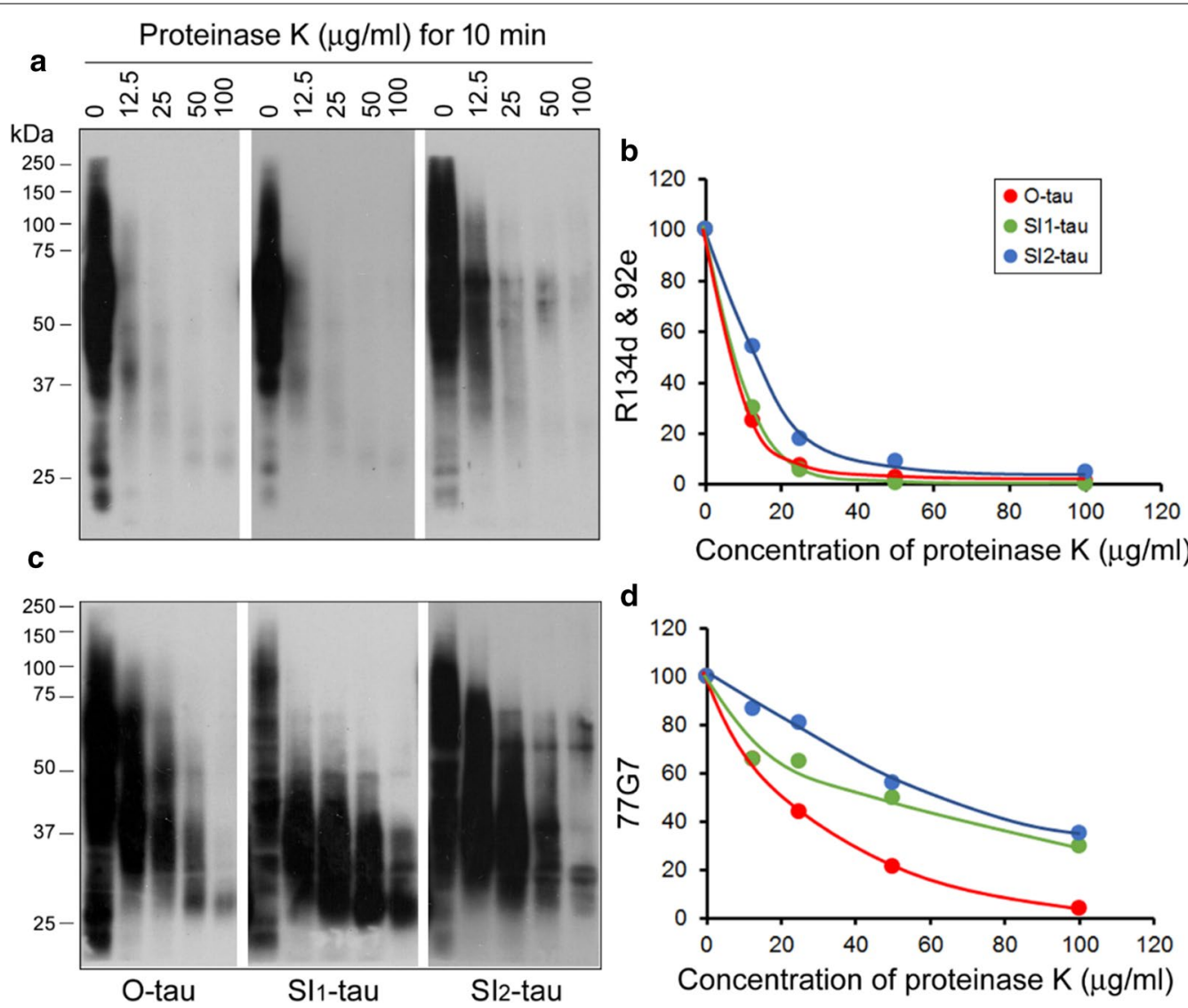

d

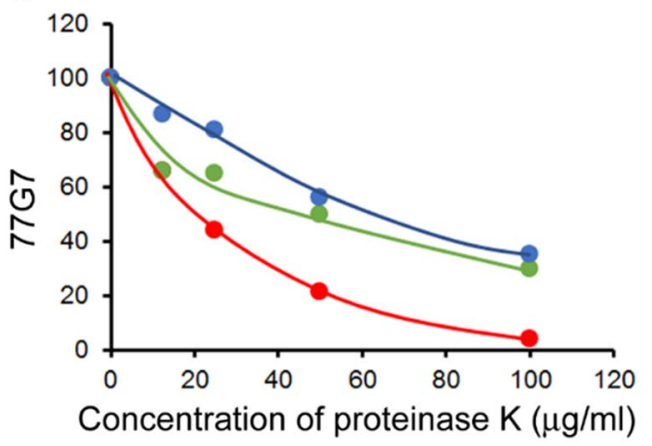

Fig. 4 AD tau fractions are differentially resistant to proteinase $K$. AD tau fractions were incubated with various concentrations of proteinase $K$. The proteolyzed products were analyzed by Western blots developed with a mixture of R134d and 92e (a) and with $77 G 7$ (c). The level of tau was quantified densitometrically and plotted against proteinase $\mathrm{K}$ concentration $(\mathbf{b}, \mathbf{d})$

proteinase $\mathrm{K}$ were observed in the blots developed with R134d/92e than with $77 G 7$ (Fig. 4b, d), suggesting that the microtubule-binding domain is relatively resistant to proteinase K. R134d/92e blot revealed that $\mathrm{SI}_{2}$-tau was decreased less rapidly than O-tau and $\mathrm{SI}_{1}$-tau (Fig. 4a, b), and 77G7 blots showed greater and faster reduction of $\mathrm{O}$-tau than did $\mathrm{SI}_{1}$-tau and $\mathrm{SI}_{2}$-tau by proteinase $\mathrm{K}$ digestion (Fig. 4c, d). Both R134d/92e and 77G7 blots showed proteinase $\mathrm{K}$-resistant 55 - to $65-\mathrm{kDa}$ tau in $\mathrm{SI}_{2}$-tau, but not in other fractions. Taken together, these results suggest that the amount of resistance to proteinase $\mathrm{K}$ was $\mathrm{O}$-tau $<\mathrm{SI}_{1}$-tau $<\mathrm{SI}_{2}$-tau.

\section{AD tau fractions capture tau differentially}

Aggregated and misfolded protein is able to recruit and to template the protein in normal conformation to the misfolded conformation, termed as the prionlike properties [20]. To determine the ability of AD tau fractions in recruiting tau, we performed overlay assay as reported recently [26]. We overexpressed tau ${ }_{151-391}$ tagged with HA in HEK-293FT cells. The crude extract of HEK-293/tau ${ }_{151-391}$ was used in tau capture assay (Fig. 5a). Tau ${ }_{151-391}$ comprises the $\beta$-sheet-forming core of the PHF structure. We dotted O-tau, $\mathrm{SI}_{1}$-tau, $\mathrm{SI}_{2}$-tau, and HS-tau with similar levels of tau on NC membrane. One membrane was incubated with the mixture of R134d and 92e to detect the levels of tau in the fractions. Another membrane was subjected to overlay assay. The membrane was incubated with HEK/ $\operatorname{tau}_{151-391}$ extract after blocking. The captured tau was analyzed by anti-HA followed by HRP- $2^{\text {nd }}$ antibody and ECL. The overlay assay revealed anti-HA immunoactivity in the membrane dotted with $\mathrm{O}$-tau and $\mathrm{SI}_{1}$-tau, but not with $\mathrm{SI}_{2}$-tau and HS-tau, in a dose-dependent manner (Fig. 5b, d), indicating capture of tau ${ }_{151-391}$ by O-tau and $\mathrm{SI}_{1}$-tau, but not $\mathrm{SI}_{2}$-tau or HS-tau.

To further confirm capture of tau $\mathrm{ta1}_{151-391}$ by O-tau and $\mathrm{SI}_{1}$-tau, but not by $\mathrm{SI}_{2}$-tau, three sets of O-tau, $\mathrm{SI}_{1}$-tau, and $\mathrm{SI}_{2}$-tau from three $\mathrm{AD}$ brains were prepared and subjected to the overlay assay (Fig. 5a). Again, we found that $\mathrm{O}$-tau and $\mathrm{SI}_{1}$-tau were able, but $\mathrm{SI}_{2}$-tau was 
a
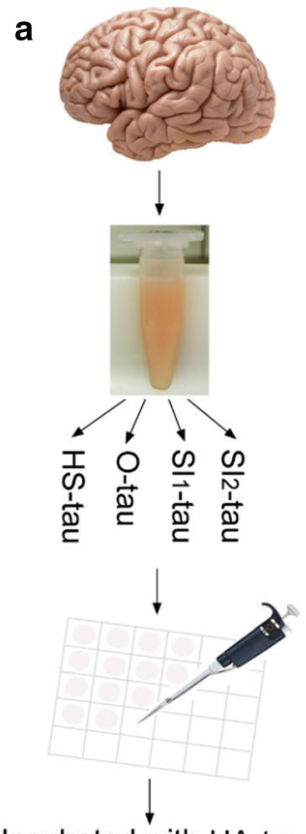

Incubated with HA-tau151-391

d

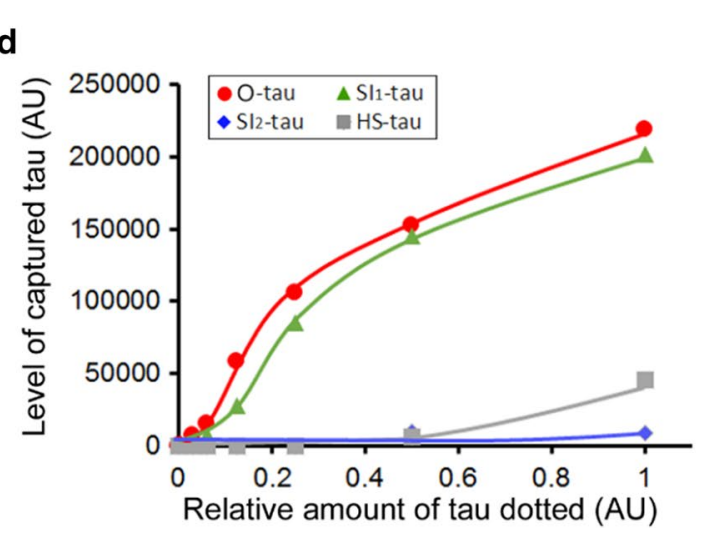

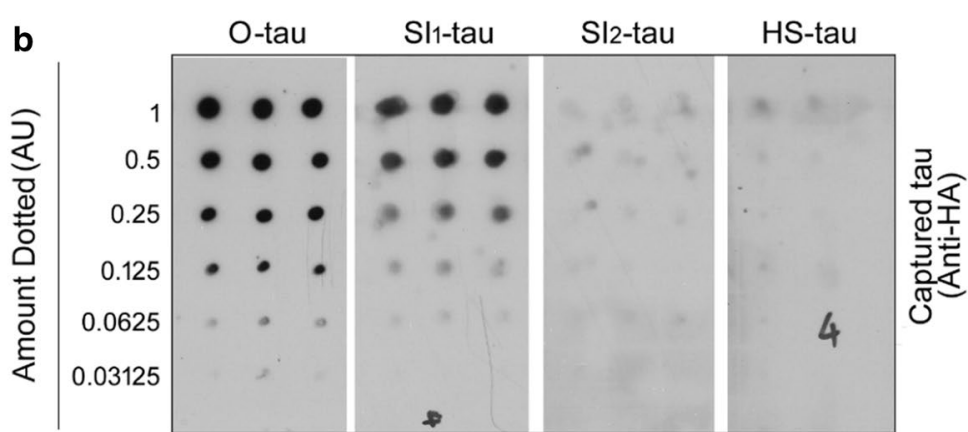

C

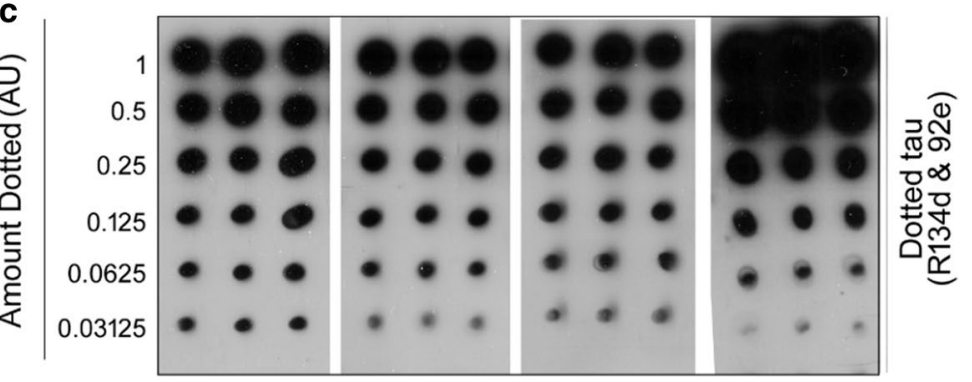

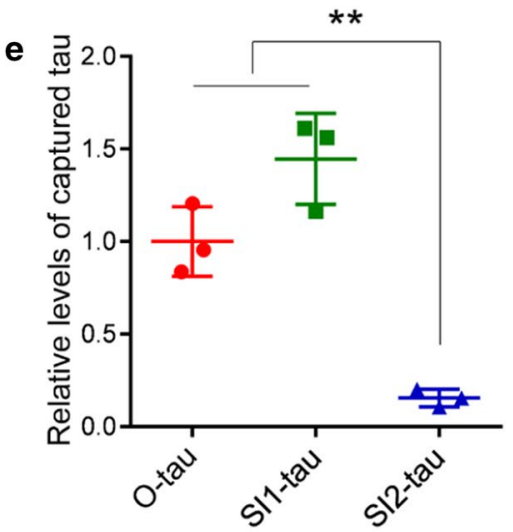

Fig. 5 AD tau fractions capture tau differentially. a Schematic of tau capture assay. $\mathbf{b}$ Various mounts of AD tau fractions were dotted onto NC membrane. After blocking, the membranes were incubated with cell extract containing HA-tau ${ }_{151-391}$, and the captured tau was detected by anti-HA, followed by HRP-2 ${ }^{\text {nd }}$ antibody and ECL. c Levels of tau in the fractions were analyzed by immuno-dot-blots developed with the mixture of R134d and 92e. $\mathbf{d}$ Levels of tau captured by the four tau fractions derived from the same AD cerebral cortex were plotted against the level of the dotted tau. e Levels of tau captured by O-tau, $\mathrm{SI}_{1}$-tau, and $\mathrm{SI}_{2}$-tau from three AD brains. The data are presented as mean $\pm S D$. ${ }^{* *} \mathrm{p}<0.05$

unable, to capture tau ${ }_{151-391}$ from the cell extract consistently (Fig. 5e).

\section{AD tau fractions seed tau aggregation in cultured cells differentially}

Seeding tau aggregation by misfolded tau in cultured cells is the basis of prion-like activity. We recently reported deletion of the first 150 and the last 50 amino acid (a.a.) of tau enhanced its aggregation seeded by AD O-tau [26]. To determine the seeding activity of these $\mathrm{AD}$ tau fractions, we overexpressed $\mathrm{HA}-\mathrm{tau}_{151-391}$ in HeLa cells and treated the cells with O-tau, $\mathrm{SI}_{1}$-tau,
$\mathrm{SI}_{2}$-tau, or HS-tau containing similar tau levels for $42 \mathrm{~h}$ after $6 \mathrm{~h}$ transfection. The cells were immuno-stained with anti-HA, and the numbers of cells with aggregates were counted. No aggregated HA-tau 151-391 $_{\text {was }}$ observed in the cells without treatment with $\mathrm{AD}$ tau fractions (Fig. 6a, b). O-tau treatment induced $\sim 25 \%$ cells with tau aggregates, and $\mathrm{SI}_{1}$-tau induced $\sim 15 \%$ cells with aggregation. Significantly fewer cells were induced to form aggregates by $\mathrm{SI}_{2}$-tau, and no significant tau aggregates were formed by HS-tau (Fig. 6a, b). Thus, these results suggest the strongest seeding activity of O-tau. The seeding activity of AD tau fractions 


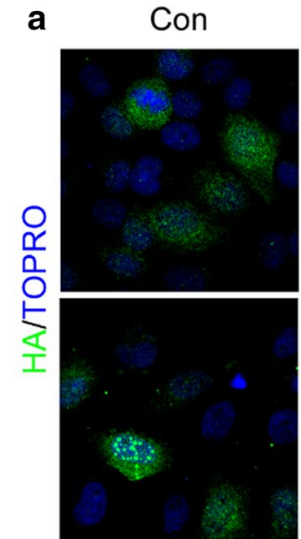

Sl2-tau
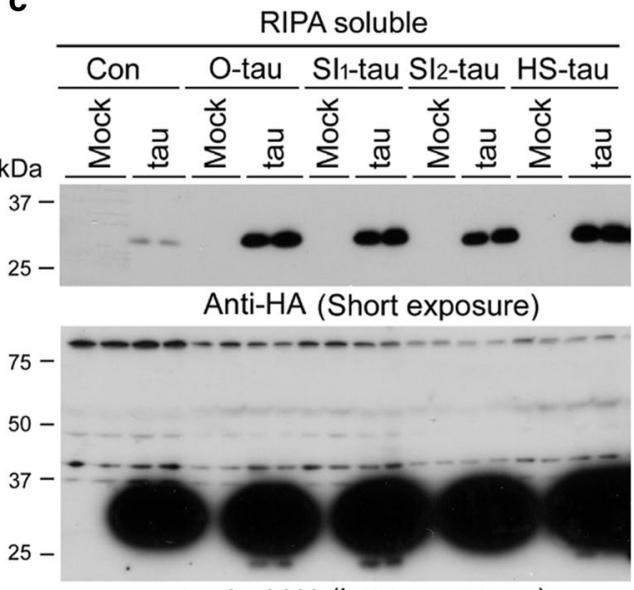

Anti-HA (Long exposure)

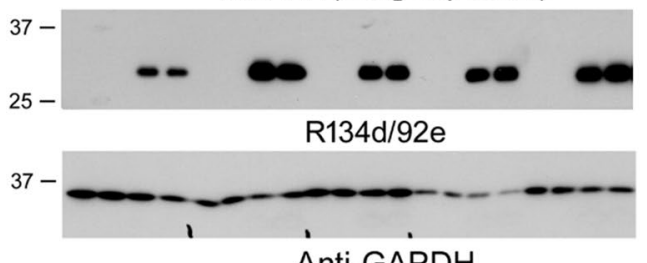

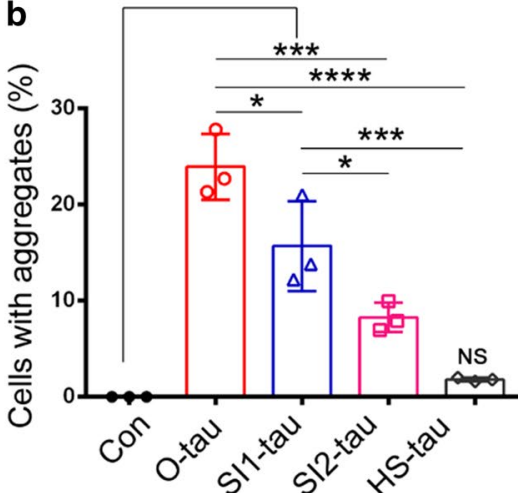

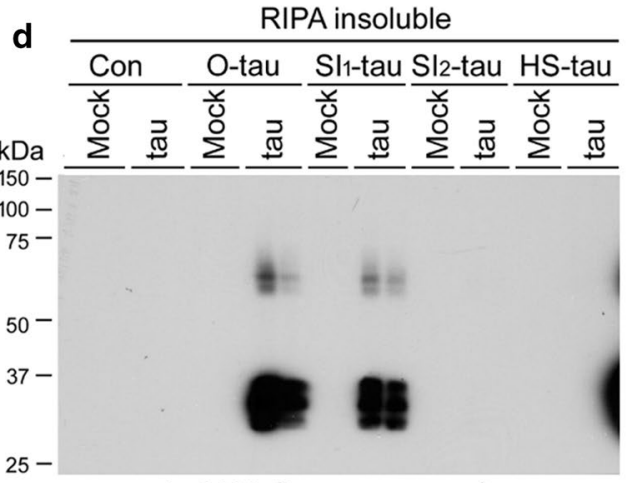

Anti-HA (Long exposure)

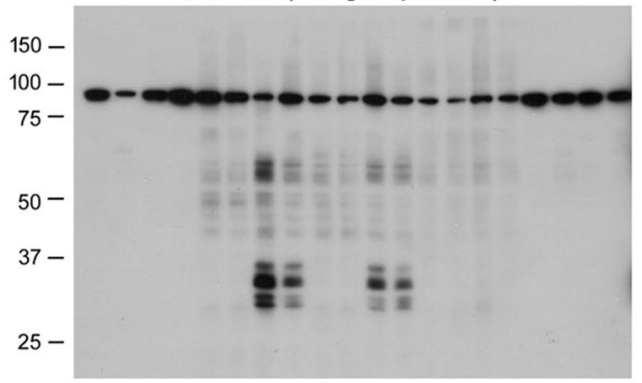

$\mathrm{R} 134 \mathrm{~d} / 92 \mathrm{e}$

Fig. 6 AD tau fractions template tau aggregation in cultured cells distinctively. $\mathbf{a}$, $\mathbf{b}$ HeLa cells were transfected with $p C l / H A-t a u_{151-391}$ and treated with AD tau fractions containing similar tau levels. The cells were immunostained with anti-HA, followed by Alex488-Anti-mouse IgG. The numbers of cells with tau aggregation were counted. The data on the percentage of cells with aggregation are presented as mean $\pm S D$. ${ }^{*} p<0.05$, ${ }^{* * *} \mathrm{p}<0.001,{ }^{* * *} \mathrm{p}<0.0001$. c, d HEK-293FT/HA-tau ${ }_{151-391}$ cells treated with AD tau fractions containing similar tau levels. The cells were lysed in RIPA buffer $42 \mathrm{~h}$ after treatment. RIPA-soluble (c) and -insoluble (d) taus were analyzed by Western blots developed with anti-HA and a mixture of R134d and $92 \mathrm{e}$

was reduced gradually from $\mathrm{O}$-tau, $\mathrm{SI}_{1}$-tau, $\mathrm{SI}_{2}$-tau, to HS-tau.

To analyze biochemically the tau aggregation induced by the AD tau fractions, we also lysed the cells with RIPA buffer and analyzed the levels of tau in RIPA-soluble and -insoluble fractions by Western blots developed with anti-HA and a mixture of R134d and 92e. Anti-HA blots revealed no soluble and insoluble tau in the mock cells treated with AD tau fractions (Fig. 6c, d). The lev-

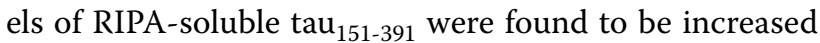
in cells treated with the tau fractions (Fig. 6c). A significant amount of RIPA-insoluble tau was found in HEK293FT/HA-tau ${ }_{151-391}$ cells treated with O-tau and $\mathrm{SI}_{1}$-tau, but not in cells treated with $\mathrm{SI}_{2}$-tau or HS-tau (Fig. 6d). We found only one $28-\mathrm{kDa}$ band in RIPA-soluble fraction, but three major bands in RIPA-insoluble fraction 
in cells treated with $\mathrm{O}$-tau and $\mathrm{SI}_{1}$-tau, which suggested hyperphosphorylation of RIPA-insoluble tau. In addition to $\sim 28$ - to $32-\mathrm{kDa}$ tau, we found 60 - to $65-\mathrm{kDa}$ SDS - and $\beta$-ME-resistant HMW-tau in RIPA-insoluble factions in O-tau- and $\mathrm{SI}_{1}$-tau-treated cells (Fig. 6d), but not in corresponding RIPA-soluble factions (Fig. 6c). R134d/92e blots showed immunoactivity in tau strain-treated mock cells, but clearly more tau in HEK-293FT/HA-tau ${ }_{151-391}$ cells treated with $\mathrm{O}$-tau and $\mathrm{SI}_{1}$-tau, compared with control treatment (Fig. 6d). Taken together, these results suggest that $\mathrm{O}$-tau and $\mathrm{SI}_{1}$-tau, but not $\mathrm{SI}_{2}$-tau and HS-tau, have prion-like properties to seed tau aggregation in cultured cells.

\section{Heat treatment does not passivate the prion-like activity of pathological tau}

HS-tau derived from $235,000 \times g$ supernatant (Sup-tau) of brain homogenate by heat treatment (Fig. 1) did not show prion-like activities (Figs. 5, 6). HS-tau was less truncated and least phosphorylated. We found that both Sup-tau and HS-tau were almost similar in Western blots developed with polyclonal pan-tau antibodies (Fig. 7a), monoclonal antibodies against specific epitopes (Fig. 7b), and site-specific and phosphorylation-dependent tau antibodies (Fig. 7c), suggesting that heat treatment did not affect the truncation and phosphorylation of tau.

Next, we performed overlay assay as described above to compare the ability of Sup-tau with HS-tau to recruit tau. We found that consistently HS-tau could not capture tau, but Sup-tau was able to recruit tau from HEK-293FT/ tau $_{151-391}$ cell extract (Fig. $7 d$, e), when even a smaller amount of tau in Sup-fraction was applied (Fig. 7d, e). These results suggest that either heat treatment inactivates tau's ability to bind to tau ${ }_{151-391}$ or the Sup-tau might contain the small-size oligomers. It is well known that heat treatment removes aggregated tau [48]. Thus, unlike HS-tau, Sup-tau might contain small-size oligomeric tau.

We then studied whether heat treatment kills the prion-like activity of pathological tau. We dotted various amounts of O-tau on NC-membranes parallelly. One set of membranes was boiled in $50 \mathrm{mM}$ Tris- $\mathrm{HCl}, 0.75 \mathrm{M}$ $\mathrm{NaCl}$, for $10 \mathrm{~min}$ to mimic heat treatment as the HS-tau preparation. Another membrane was incubated with the same buffer at RT as control treatment. Then, the membranes were overlaid with the HEK-293FT/tau ${ }_{151-391}$ cell extract for tau capture assay or with a mixture of R134d and 92e for tau assay, as described above. We found that heat treatment caused a slight reduction of tau level (Fig. 7f, g). Similarly, a slightly lesser amount of tau was captured by heat-treated O-tau than by control O-tau (Fig. 7f, g), suggesting that the heat treatment did not affect the prion-like activity of O-tau.
$A D$ tau fractions induce tau pathology in vivo differentially

To determine the seeding activity of $\mathrm{AD}$ tau fractions in vivo, we injected the four $\mathrm{AD}$ tau fractions with similar tau levels into the hippocampus in 18-month-old FVB mice and analyzed tau pathology in ipsilateral and contralateral hippocampi by immunofluorescence staining with AT8 3 months after injection. We found many AT8-positive neurons in both the ipsilateral and contralateral CA1 (Fig. 8a) and the ipsilateral CA3 (Fig. 8b) of the hippocampi of mice injected with $\mathrm{O}$-tau, and a few AT8-positive neurons in the ipsilateral CA1 (Fig. 8a) of $\mathrm{SI}_{1}$-tau-injected mice. No obvious AT8 immunoactivity was observed in the ipsilateral and contralateral hippocampi of $\mathrm{SI}_{2}$-tau- or HS-tau-injected mice. AT8 immunostaining was not detectable in the cortex of the mice injected with any of the four tau fractions. AT8 immunostaining was dramatically higher in both the ipsilateral and contralateral hippocampi injected with O-tau than in those injected with $\mathrm{SI}_{1}$-tau, $\mathrm{SI}_{2}$-tau, or HS-tau (Fig. 8c), suggesting that $\mathrm{O}$-tau serves as potent seeds to induce tau pathology in vivo.

\section{Discussion}

Tau in $\mathrm{AD}$ brain exists in monomeric, oligomeric, and fibric forms. In the present study, we isolated from $\mathrm{AD}$ brain four fractions-O-tau, $\mathrm{SI}_{1}$-tau, $\mathrm{SI}_{2}$-tau, and HStau-and analyzed their biochemical and prion-like properties. We found that $\mathrm{O}$-tau, $\mathrm{SI}_{1}$-tau, and $\mathrm{SI}_{2}$-tau, but not HS-tau, contained SDS- and reducing agentresistant HMW-tau. O-tau and $\mathrm{SI}_{2}$-tau revealed similar patterns of truncation and hyperphosphorylation. Compared to $\mathrm{O}$-tau and $\mathrm{SI}_{1}$-tau, $\mathrm{SI}_{2}$-tau was more resistant to proteolysis by proteinase $\mathrm{K}$. O-tau, $\mathrm{SI}_{1}$-tau, but not $\mathrm{SI}_{2}$-tau or HS-tau, captured/sequestered tau in vitro and templated tau aggregation in cultured cells. Heat treatment did not inactivate the prion-like activity of O-tau. O-tau induced tau pathology in the ipsilateral and contralateral hippocampi, $\mathrm{SI}_{1}$-tau only induced it in the ipsilateral hippocampus, and $\mathrm{SI}_{2}$-tau or HS-tau could not induce tau aggregation in the hippocampi of 18-monthold FVB wild-type mice, as determined 3 months after injection. These results suggest that tau in the four isolated fractions showed distinct biochemical and prionlike properties. O-tau and $\mathrm{SI}_{2}$-tau showed similarities in hyperphosphorylation and truncation. Oligomeric O-tau and $\mathrm{SI}_{1}$-tau in $\mathrm{AD}$ brain may serve as seeds to induce tau aggregation. Monomeric tau and $\mathrm{SI}_{2}$-tau were inert in the prion-like properties, and they could not induce tau pathogenesis.

Tau is truncated at multiple sites in AD brain [49, 54]. Truncation may facilitate tau pathogenesis. We found in a parallel study that deletion of the first 150 or 230 a.a. and the last 50 a.a. enhanced tau's site-specific 

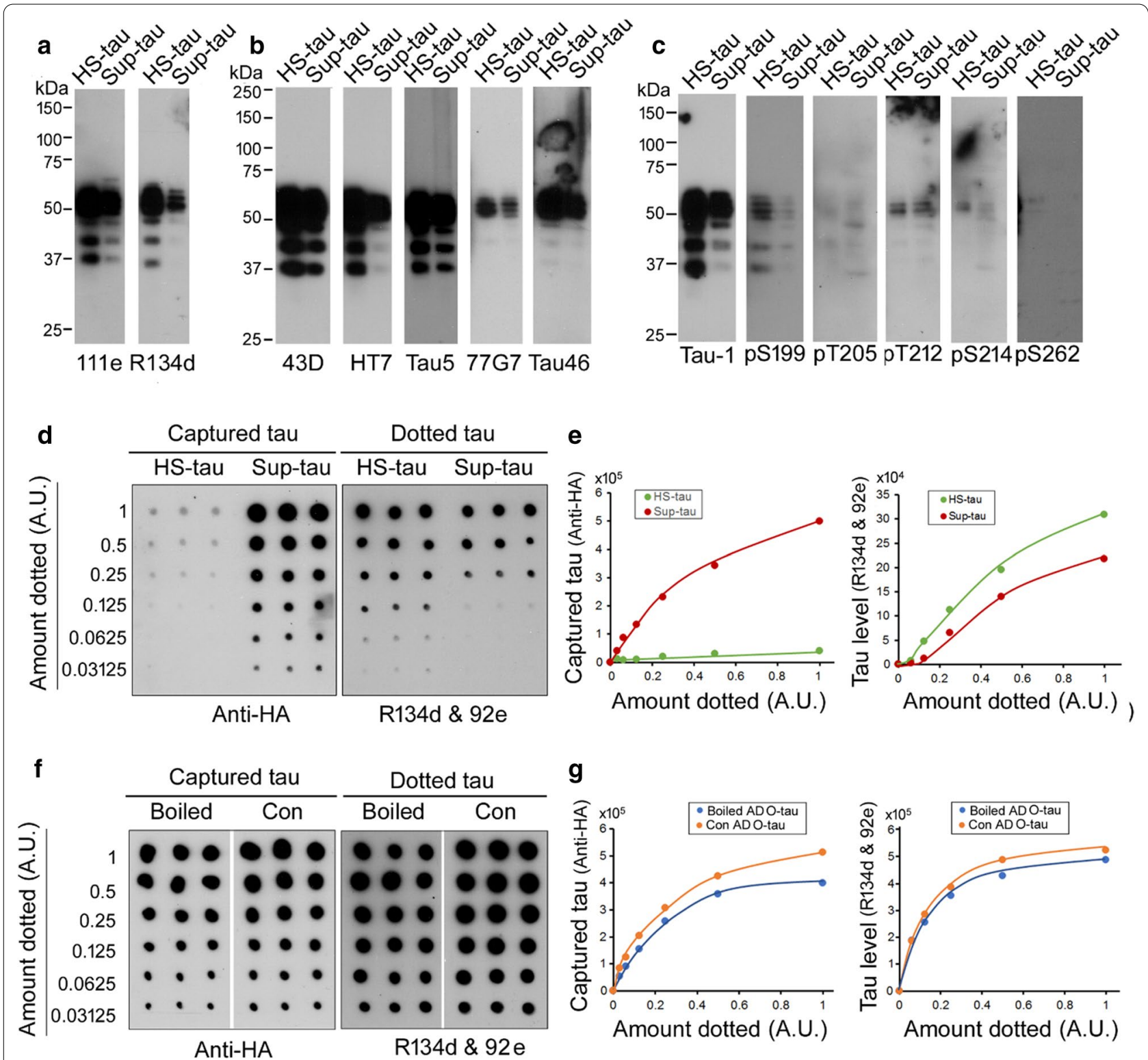

Fig. 7 Heat treatment does not passivate the prion-like activity of O-tau. a-c Sup-tau and HS-tau were similar in phosphorylation and truncation. Sup-tau and HS-tau were analyzed by Western blots developed with polyclonal tau antibodies (a), monoclonal tau antibodies (b), and site-specific and phosphorylation-dependent antibodies (c). d, e Sup-tau, but not HS-tau, was able to capture tau. Various amount of Sup-tau and HS-tau were applied on NC membranes. One membrane was subjected to tau overlay assay by sequential incubation of HEK-293FT/HA-tau $151-391$ Cell extract, anti-HA (d, left), and one membrane was subjected to analysis of tau level by dot-blot developed with a mixture of R134d and 92e (d, right). The captured tau (e, left) and the dotted tau levels (e, right) detected in panel d were plotted against applied Sup-tau and HS-tau. $\mathbf{f}$, $\mathbf{g}$ Heat treatment did not affect O-tau to recruit tau. Various amounts of O-tau were applied onto NC membrane. One membrane was boiled, and the other membrane was treated with the same buffer at RT as a control. The membranes were incubated with cell extract containing HA-tau ${ }_{151-391}$. The captured tau (f, left) and applied tau (f, right) were analyzed as described above and plotted with the levels of O-tau (g). A.U., arbitrary units

hyperphosphorylation and self-aggregation as well as its binding to, and its aggregation seeded by, O-tau. $\operatorname{tau}_{151-391}$, corresponding to the $\beta$-sheet-forming core of the PHF structure [46, 55], contains microtubulebinding repeats and showed the highest pathologyassociated activities. The microtubule-binding repeats of tau (tauRD) with P301S mutation have been used in HEK293-tau-biosensor cells for tau-seeding [57]. $\mathrm{Tau}_{151-391}$ aggregates induced by $\mathrm{O}$-tau were thioflavin $\mathrm{T}$-positive and showed SDS- and $\beta$-ME-resistant HMW-tau in Western blots. By using RIPA buffer, aggregated tau was yielded in RIPA-insoluble fraction. 

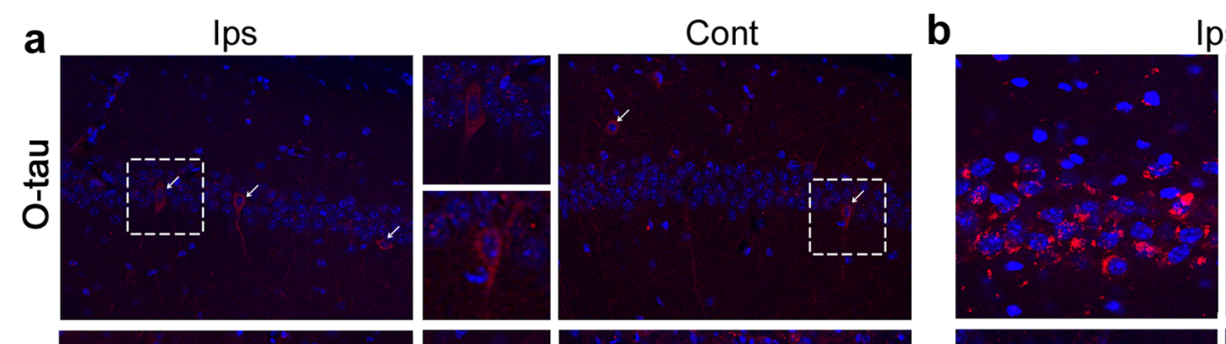

Ips
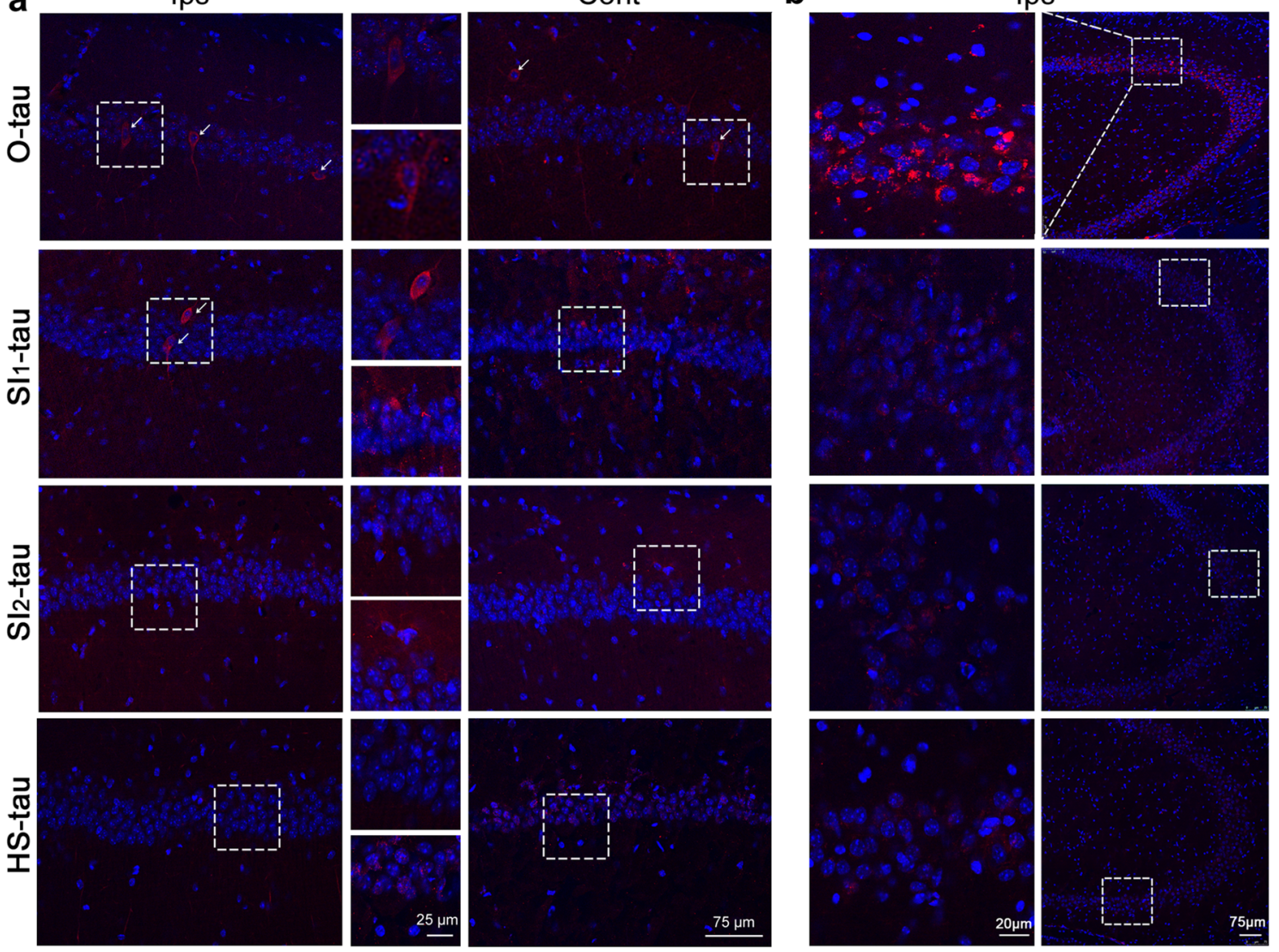

C

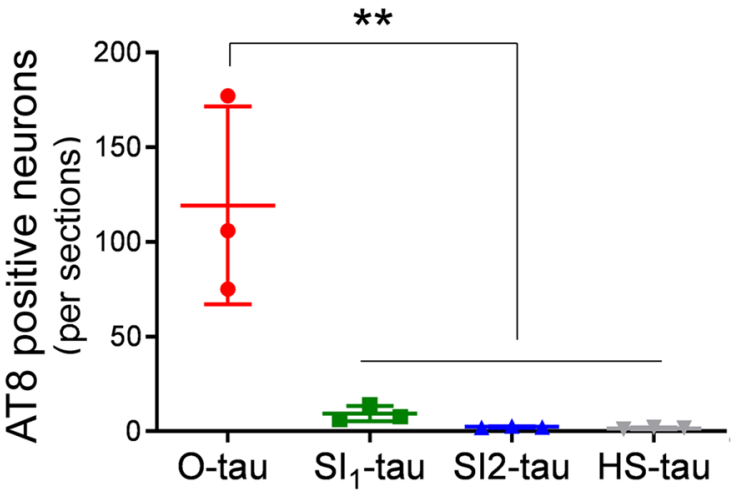

IPS

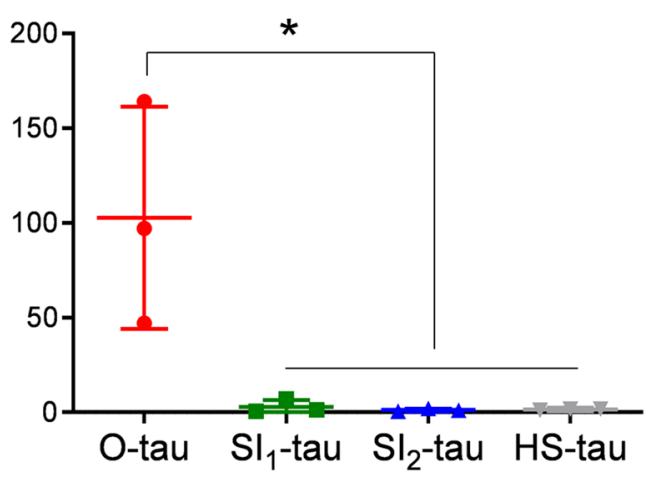

\section{Cont}

Fig. 8 AD tau fractions induce tau pathology differentially in vivo. a, b AD tau fractions at similar tau levels were injected into hippocampus of 18-month-old-FVB mice. Brain sections were immunostained with AT8 3 months after injection. Hoechst dye was used to stain nuclei. Representative AT8 immunostaining of ipsilateral (Ips) and contralateral (Cont) CA1s (a) and Ips CA3 (b) of the mouse hippocampus after injection with AD tau fractions. c AT8-positive neurons in Ips hippocampus (left) and Cont hippocampus (right) were quantified. Data are presented as mean $\pm S D$, **P $<0.05 ;{ }^{* *} \mathrm{P}<0.01$

O-tau yielded from $27,000 \times g$ to $235,000 \times g$ fraction of AD brain homogenate [39]. Different from the tau in the $235,000 \times g$ supernatant, O-tau was abnormally hyperphosphorylated and formed SDS- and
$\beta$-ME-resistant HMW aggregates, which lacked the $\mathrm{N}$-terminal portion. $\mathrm{O}$-tau displayed very potent prionlike activities, capturing/sequestering tau and seeding tau aggregation in cultured cells and in vivo. Heat 
treatment removes aggregated tau from the supernatant [48]. We found that heat treatment did not change O-tau ability to capture tau. However, HS-tau derived from Sup-tau could not capture tau, suggesting that aggregated tau, but not monomeric tau has prion-like activity. It was reported that tau trimers are the minimal propagation unit spontaneously internalized to seed intracellular aggregation [44]. However, large ( $>10$ mer) aggregated tau, but not small, oligomeric ( $<6$ mer) tau, from P301S transgenic mouse brains seeded cellular tau aggregation [34]. Similarly, we previously found that compared with O-tau from AD brain, O-tau from $3 x \mathrm{Tg}-\mathrm{AD}$ mouse brain showed much weaker seeding activity [40]. Furthermore, tau monomer purified from AD brain also had intrinsic seeding activity, and selfassociated to produce larger seed-competent assemblies. It was proposed that tau monomer occupies two distinct and stable conformational ensembles: inert and seeding-competent [45]. Thus, we speculate that the $235,000 \times g$ supernatant contains small aggregates or/ and seeding-competent tau monomer that may not be SDS- and $\beta$-ME-resistant but could capture tau and heat-treatment removes both species of tau.

Similarly, serial sedimentation can divide tau from $\mathrm{AD}$ brain into various fractions [53]. It was found that $3,000 \times g$ and $10,000 \times g$ AD brain extracts, which presumably contained HMW proteins, could be up-taken by cultured neurons, but $50,000 \times g$ and $150,000 \times g$ extracts, from which HMW tau was depleted by sedimentation, could not be up-taken by neurons [53]. The $3,000 \times g$ brain extracts showed significantly higher seeding activity than $150,000 \times g$ extracts. Thus, the $3,000 \times g$ and $10,000 \times g$ extracts contain various sizes of oligomeric tau [53], which may serve as predominant seeds to template tau aggregation. Consistently, we found here that O-tau from $27,000 \times g$ to $235,000 \times g$ displayed the prion-like activities.

A previous study showed that $\mathrm{AD}$ tau from the $10,000 \times g$ brain homogenate in $0.1 \%$ sarkosyl-high salt buffer to $235,000 \times g$ in $1 \%$ sarkosyl induced tau aggregation in vitro and in vivo [28]. Different from this protocol, we first separated monomeric and oligomeric tau from aggregated tau by centrifugation of brain homogenate at $27,000 \times g$ and then incubated the pellet in the buffer containing $0.1 \%$ sarkosyl and centrifuged at $10,000 \times g$. The supernatant and the pellet probably contain loose and compressed tau aggregates, respectively. These fractions were incubated in $1 \%$ sarkosyl containing buffer and centrifuged at $100,000 \times g$ to yield $\mathrm{SI}_{1}$-tau and $\mathrm{SI}_{2}$-tau. Both $\mathrm{SI}_{1}$ and $\mathrm{SI}_{2}$ fractions contained SDS- and $\beta$-ME-resistant HMW-tau, which did not react with $\mathrm{N}$-terminal tau antibodies and was hyperphosphorylated at multiple sites. A relatively higher level of tau was detected in the
$\mathrm{SI}_{1}$ fraction by the $\mathrm{N}$-terminal antibodies than in the $\mathrm{SI}_{2}$ fraction. Compared to O-tau, less tau was detected by the antibodies against microtubule-binding repeats, suggesting that $\mathrm{SI}_{1}$-tau and $\mathrm{SI}_{2}$-tau may be less accessible to these antibodies. Misfolded protein aggregates are usually resistant to proteolysis. We found that the resistance of O-tau, $\mathrm{SI}_{1}$-tau, and $\mathrm{SI}_{2}$-tau to proteinase $\mathrm{K}$ digestion was increased. Most interestingly, $\mathrm{SI}_{1}$-tau was able to capture tau and to seed tau aggregation in cultured cells in prion-like fashion, but $\mathrm{SI}_{2}$-tau was inert in these prionlike properties.

Prion-like spread of misfolded tau aggregates might underlie the stereotypic progression of neurodegenerative tauopathies. We reported in 1994 that the cytosolic and hyperphosphorylated tau from AD brain, named AD p-tau, sequestered tau and induced tau aggregation in vitro, which is the first study showing the prion-like activity of AD p-tau [3, 4]. AD p-tau was further purified by ion-exchange chromatography to remove nonhyperphosphorylated tau in O-tau [39]. Different from AD p-tau, PHF-tau could not sequester normal tau [4]. In the present study, we found that $\mathrm{O}$-tau and $\mathrm{SI}_{1}$-tau, but not $\mathrm{SI}_{2}$-tau, captured tau and templated tau aggregation. We speculate that the major component of $\mathrm{SI}_{2}$-tau may be PHF-tau.

Tau pathology initiates in the subcortical regions, transentorhinal cortex, and entorhinal cortex (stages I and II), then appears in the hippocampal formation and some parts of the neocortex (stages III and IV), followed by most of the neocortex (stages V and VI) $[7,10]$. Immunohistochemical (IHC) study with AT8, a principal tool to define AD intraneuronal pathology [43], showed that AT8 signal first appears in the locus coeruleus (LC), suggesting that tau aggregation in the $\mathrm{LC}$ may represent the earliest phase of $\mathrm{AD}$ pathogenesis $[9,11]$. Individuals at stages I and II are asymptomatic, but over half of the subjects at NFT stages III-IV exhibited signs of mild cognitive impairment, and over $90 \%$ of subjects at NFT stages V-VI showed moderate to severe dementia [31]. By using HEK293-tau-biosensor cells, tau-seeding activity from AD brains correlates positively with Braak stage and negatively with MMSE [19], but pathological seeding activity begins in the transentorhinal/entorhinal cortices (TRE/ EC) rather than in the LC [36]. In the present study, we analyzed various tau fractions isolated from $A D$ cerebral cortex at NFT stages V-VI and found that various AD tau fractions displayed different biochemical and prion-like properties, indicating the heterogenicity of pathological activity of tau fractions in AD brains.

In addition to $\mathrm{AD}$, aggregated tau is a common feature of tauopathies. Distinct tau fractions from various tauopathies have been shown to induce distinct tau aggregation in mouse brains [50]. Thus, heterogeneity 


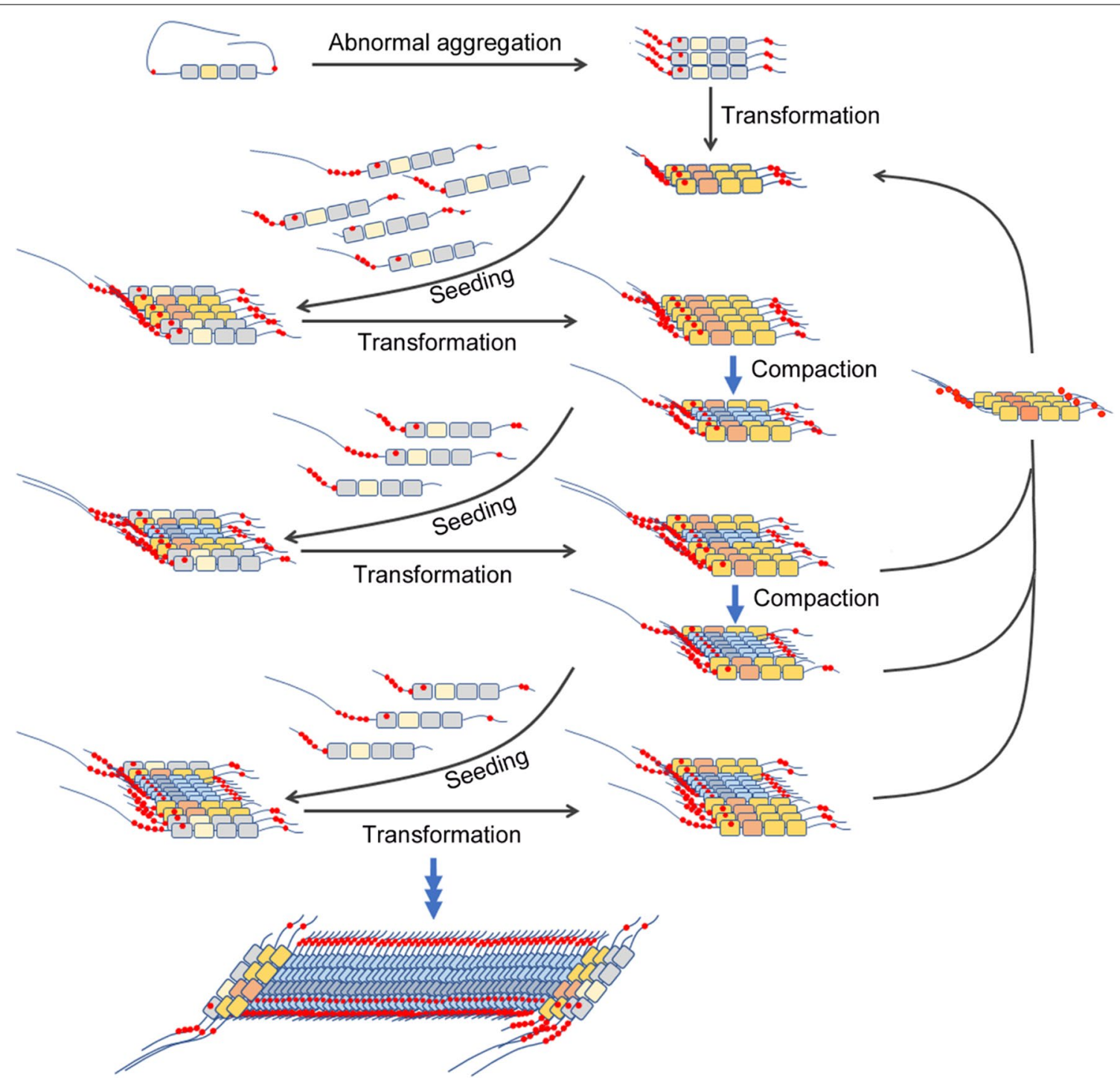

Fig. 9 Proposed working model of the prion-like propagation of tau. Abnormally aggregated tau forms O-tau, which recruits tau and templates its transformation. Inner aggregates of tau are compacted to form compressed NFTs, $\mathrm{SI}_{2}$-tau, in which the prion-like activities are passivated. Outer aggregates of tau, $\mathrm{SI}_{1}$-tau, on one hand recruit normal tau and template the transformation, resulting in the growth of the NFTs. On the other hand, these aggregates may be released to form O-tau, which seeds to form new aggregation in the cells or in new cells

of tau pathology both within AD and among different tauopathies could be due to different fractions with different biological and prion-like properties. This heterogeneity poses a major challenge in targeting tau for development of effective therapeutic treatment for tauopathies.

Taken together, we propose a working model of tau propagation (Fig. 9) in which O-tau recruits normal tau and templates the recruited tau and transforms to $\beta$-sheet conformation, resulting in loose aggregates, which recruit and template normal tau transformation. During the progression of tau pathogenesis, inner aggregated tau is truncated and condensed to form compressed aggregates, resulting in loss of its prion-like activities. Based on this model, we speculate that O-tau may initiate tau aggregation, but $\mathrm{SI}_{1}$-tau may contribute to the growth of aggregated tau.

\section{Acknowledgements}

We are thankful to Dr. Peter Davies for his generous gift of tau monoclonal antibody PHF-1 and to Dr. Lester I. Binder for Tau-1 and to Ms. Maureen Marlow for copy-editing the manuscript. This work was supported in part by funds from the New York State Office for People With Developmental Disabilities, Nantong University, and the Neural Regeneration Co-innovation Center of Jiangsu Province and by grants from the U.S. Alzheimer's Association (DSAD15-363172) and the Postgraduate Research \& Practice Innovation Program of Jiangsu Province (KYCX18_2413). 


\section{Authors' contributions}

L.L., R.S, J.G., Y.C.T., N.J., K.D., and J.X. carried out the study in in vitro and in cultured cells. L.L, Y.Z., D.Z., R.W., and N.J. treated the animals and performed immunohistochemical studies. D.C., C.X.G., and K.I helped in discussing the data and editing the paper. F.L. conceived, designed, and supervised the study and wrote the paper.

\section{Availability of data and materials}

The datasets generated and/or analyzed during the present study are available from the corresponding author, Dr. Fei Liu, upon reasonable request.

\section{Ethics approval and consent to participate}

All procedures involving mice were reviewed and approved by our Institutional Animal Care and Use Committee and were carried out according to guidelines of the National Institutes of Health. The use of autopsied frozen human brain tissue was in accordance with the National Institutes of Health guidelines and was exempted by the Institutional Review Board (IRB) of the New York State Institute for Basic Research in Developmental Disabilities because "the research does not involve intervention or interaction with the individuals" nor "is the information individually identifiable".

\section{Competing interests}

The authors declare that they have no competing interests.

\section{Author details}

${ }^{1}$ Department of Neurochemistry, Inge Grundke-lqbal Research Floor, New York State Institute for Basic Research in Developmental Disabilities, 1050 Forest Hill Road, Staten Island, NY 10314, USA. ${ }^{2}$ Key Laboratory of Neuroregeneration of Jiangsu and Ministry of Education of China, Co-Innovation Center of Neuroregeneration, 19 Qixiu Road, Nantong 226001, Jiangsu, China.

Received: 3 February 2021 Accepted: 4 February 2021

Published online: 17 February 2021

\section{References}

1. Ahmed Z, Cooper J, Murray TK, Garn K, McNaughton E, Clarke H, Parhizkar S, Ward MA, Cavallini A, Jackson S, Bose S, Clavaguera F, Tolnay M, Lavenir I, Goedert M, Hutton ML, O'Neill MJ (2014) A novel in vivo model of tau propagation with rapid and progressive neurofibrillary tangle pathology: the pattern of spread is determined by connectivity, not proximity. Acta Neuropathol 127(5):667-683

2. Alafuzoff I, Adolfsson R, Grundke-lqbal I, Winblad B (1987) Blood-brain barrier in Alzheimer dementia and in non-demented elderly. An immunocytochemical study Acta Neuropathol 73(2):160-166

3. Alonso AC, Zaidi T, Grundke-lqbal I, lqbal K (1994) Role of abnormally phosphorylated tau in the breakdown of microtubules in Alzheimer disease. Proc Natl Acad Sci U S A 91(12):5562-5566

4. Alonso AD, Grundke-lqbal I, labal K (1996) Alzheimer's disease hyperphosphorylated tau sequesters normal tau into tangles of filaments and disassembles microtubules. Nat Med 2(7):783-787

5. Arriagada PV, Growdon JH, Hedley-Whyte ET, Hyman BT (1992) Neurofibrillary tangles but not senile plaques parallel duration and severity of Alzheimer's disease. Neurology 42(3 Pt 1):631-639

6. Boluda S, Iba M, Zhang B, Raible KM, Lee VM, Trojanowski JQ (2015) Differential induction and spread of tau pathology in young PS19 tau transgenic mice following intracerebral injections of pathological tau from Alzheimer's disease or corticobasal degeneration brains. Acta Neuropathol 129(2):221-237

7. Braak H, Braak E (1991) Neuropathological stageing of Alzheimer-related changes. Acta Neuropathol (Berl) 82(4):239-259

8. Braak H, Braak E (1995) Staging of Alzheimer's disease-related neurofibrillary changes. Neurobiol Aging 16(3):271-278 (discussion 278-284)

9. Braak H, Del Tredici K (2011) The pathological process underlying Alzheimer's disease in individuals under thirty. Acta Neuropathol 121(2):171-181

10. Braak H, Del Tredici K (2011) Alzheimer's pathogenesis: is there neuron-toneuron propagation? Acta Neuropathol 121(5):589-595
11. Braak H, Thal DR, Ghebremedhin E, Del Tredici K (2011) Stages of the pathologic process in Alzheimer disease: age categories from 1 to 100 years. J Neuropathol Exp Neurol 70(11):960-969

12. Braak H, Feldengut S, Del Tredici K (2013) Pathogenesis and prevention of Alzheimer's disease: when and in what way does the pathological process begin? Der Nervenarzt 84(4):477-482

13. Clavaguera F, Bolmont T, Crowther RA, Abramowski D, Frank S, Probst A Fraser G, Stalder AK, Beibel M, Staufenbiel M, Jucker M, Goedert M, Tolnay M (2009) Transmission and spreading of tauopathy in transgenic mouse brain. Nat Cell Biol 11(7):909-913

14. Clavaguera F, Akatsu H, Fraser G, Crowther RA, Frank S, Hench J, Probst A, Winkler DT, Reichwald J, Staufenbiel M, Ghetti B, Goedert M, Tolnay M (2013) Brain homogenates from human tauopathies induce tau inclusions in mouse brain. Proc Natl Acad Sci U S A 110(23):9535-9540

15. de Calignon A, Polydoro M, Suarez-Calvet M, William C, Adamowicz DH, Kopeikina KJ, Pitstick R, Sahara N, Ashe KH, Carlson GA, Spires-Jones TL, Hyman BT (2012) Propagation of tau pathology in a model of early Alzheimer's disease. Neuron 73(4):685-697

16. Dujardin S, Begard S, Caillierez R, Lachaud C, Delattre L, Carrier S, Loyens A, Galas MC, Bousset L, Melki R, Auregan G, Hantraye P, Brouillet E, Buee L, Colin M (2014) Ectosomes: a new mechanism for non-exosomal secretion of tau protein. PLOS ONE 9(6):e100760

17. Fellous A, Francon J, Lennon AM, Nunez J (1977) Microtubule assembly in vitro. Purification of assembly-promoting factors. Eur J Biochem 78(1):167-174

18. Frost B, Jacks RL, Diamond MI (2009) Propagation of tau misfolding from the outside to the inside of a cell. J Biol Chem 284(19):12845-12852

19. Furman JL, Vaquer-Alicea J, White CL 3rd, Cairns NJ, Nelson PT, Diamond MI (2017) Widespread tau seeding activity at early Braak stages. Acta Neuropathol 133(1):91-100

20. Goedert M, Clavaguera F, Tolnay M (2010) The propagation of prionlike protein inclusions in neurodegenerative diseases. Trends Neurosci 33(7):317-325

21. Gong CX, Grundke-lqbal I, lqbal K (1994) Dephosphorylation of Alzheimer's disease abnormally phosphorylated tau by protein phosphatase2A. Neuroscience 61(4):765-772

22. Greenberg SG, Davies P (1990) A preparation of Alzheimer paired helical filaments that displays distinct tau proteins by polyacrylamide gel electrophoresis. Proc Natl Acad Sci U S A 87(15):5827-5831

23. Grober E, Dickson D, Sliwinski MJ, Buschke H, Katz M, Crystal H, Lipton RB (1999) Memory and mental status correlates of modified Braak staging. Neurobiol Aging 20(6):573-579

24. Grundke-lqbal I, lqbal K, Quinlan M, Tung YC, Zaidi MS, Wisniewski HM (1986) Microtubule-associated protein tau. A component of Alzheimer paired helical filaments. J. Biol. Chem. 261(13):6084-6089

25. Grundke-lqabal I, labal K, Tung YC, Quinlan M, Wisniewski HM, Binder LI (1986) Abnormal phosphorylation of the microtubule-associated protein tau (tau) in Alzheimer cytoskeletal pathology. Proc Natl Acad Sci USA 83(13):4913-4917

26. Gu J, Xu W, Jin N, Li L, Zhou Y, Chu D, Gong CX, Iqbal K, Liu F (2020) Truncation of tau selectively facilitates its pathological activities. J Biol Chem 295(40):13812-13828

27. Guo JL, Lee VM (2011) Seeding of normal Tau by pathological Tau conformers drives pathogenesis of Alzheimer-like tangles. J Biol Chem 286(17):15317-15331

28. Guo JL, Narasimhan S, Changolkar L, He Z, Stieber A, Zhang B, Gathagan RJ, Iba M, McBride JD, Trojanowski JQ, Lee VM (2016) Unique pathological tau conformers from Alzheimer's brains transmit tau pathology in nontransgenic mice. J Exp Med 213(12):2635-2654

29. Holmes BB, Furman JL, Mahan TE, Yamasaki TR, Mirbaha H, Eades WC, Belaygorod L, Cairns NJ, Holtzman DM, Diamond MI (2014) Proteopathic tau seeding predicts tauopathy in vivo. Proc Natl Acad Sci U S A 111(41):E4376-4385

30. Hu W, Zhang X, Tung YC, Xie S, Liu F, Iqbal K (2016) Hyperphosphorylation determines both the spread and the morphology of tau pathology. Alzheimers Dement 12(10):1066-1077

31. Hyman BT, Phelps CH, Beach TG, Bigio EH, Cairns NJ, Carrillo MC, Dickson DW, Duyckaerts C, Frosch MP, Masliah E, Mirra SS, Nelson PT, Schneider JA, Thal DR, Thies B, Trojanowski JQ, Vinters HV, Montine TJ (2012) National Institute on Aging-Alzheimer's Association guidelines for the 
neuropathologic assessment of Alzheimer's disease. Alzheimers Dement $8(1): 1-13$

32. Iba M, Guo JL, McBride JD, Zhang B, Trojanowski JQ, Lee VM (2013) Synthetic tau fibrils mediate transmission of neurofibrillary tangles in a transgenic mouse model of Alzheimer's-like tauopathy. J Neurosci 33(3):1024-1037

33. Iqbal K, Liu F, Gong CX (2016) Tau and neurodegenerative disease: the story so far. Nat Rev Neurol 12(1):15-27

34. Jackson SJ, Kerridge C, Cooper J, Cavallini A, Falcon B, Cella CV, Landi A, Szekeres PG, Murray TK, Ahmed Z, Goedert M, Hutton M, O'Neill MJ, Bose S (2016) Short Fibrils Constitute the Major Species of Seed-Competent Tau in the Brains of Mice Transgenic for Human P301S Tau. J Neurosci 36(3):762-772

35. Johnson KA, Schultz A, Betensky RA, Becker JA, Sepulcre J, Rentz D, Mormino E, Chhatwal J, Amariglio R, Papp K, Marshall G, Albers M, Mauro S, Pepin L, Alverio J, Judge K, Philiossaint M, Shoup T, Yokell D, Dickerson B, Gomez-Isla T, Hyman B, Vasdev N, Sperling R (2016) Tau positron emission tomographic imaging in aging and early Alzheimer disease. Ann Neurol 79(1):110-119

36. Kaufman SK, Del Tredici K, Thomas TL, Braak H, Diamond MI (2018) Tau seeding activity begins in the transentorhinal/entorhinal regions and anticipates phospho-tau pathology in Alzheimer's disease and PART. Acta Neuropathol 136(1):57-67

37. Kfoury N, Holmes BB, Jiang H, Holtzman DM, Diamond MI (2012) Transcellular propagation of Tau aggregation by fibrillar species. J Biol Chem 287(23):19440-19451

38. Khatoon S, Grundke-lqbal I, lqbal K (1992) Brain levels of microtubuleassociated protein tau are elevated in Alzheimer's disease: a radioimmuno-slot-blot assay for nanograms of the protein. J Neurochem 59(2):750-753

39. Kopke E, Tung YC, Shaikh S, Alonso AC, lqbal K, Grundke-lqbal I (1993) Microtubule-associated protein tau. Abnormal phosphorylation of a non-paired helical filament pool in Alzheimer disease. J Biol Chem 268(32):24374-24384

40. Li L, Jiang Y, Hu W, Tung YC, Dai C, Chu D, Gong CX, lqbal K, Liu F (2019) Pathological Alterations of Tau in Alzheimer's Disease and 3xTg-AD Mouse Brains. Mol Neurobiol 56(9):6168-6183

41. Liu F, Shi J, Tanimukai H, Gu J, Grundke-lqbal I, lqbal K, Gong CX (2009) Reduced O-GlcNAcylation links lower brain glucose metabolism and tau pathology in Alzheimer's disease. Brain 132(Pt 7):1820-1832

42. Liu L, Drouet V, Wu JW, Witter MP, Small SA, Clelland C, Duff K (2012) Transsynaptic spread of tau pathology in vivo. PLoS ONE 7(2):e31302

43. Mercken $M$, Vandermeeren M, Lubke U, Six J, Boons J, Van de Voorde A, Martin JJ, Gheuens J (1992) Monoclonal antibodies with selective specificity for Alzheimer Tau are directed against phosphatase-sensitive epitopes. Acta Neuropathol 84(3):265-272

44. Mirbaha H, Holmes BB, Sanders DW, Bieschke J, Diamond MI (2015) Tau trimers are the minimal propagation unit spontaneously internalized to seed intracellular aggregation. J Biol Chem 290(24):14893-14903

45. Mirbaha H, Chen D, Morazova OA, Ruff KM, Sharma AM, Liu X, Goodarzi M, Pappu RV, Colby DW, Mirzaei H, Joachimiak LA, Diamond MI (2018) Inert and seed-competent tau monomers suggest structural origins of aggregation. Elife 7:e36584

46. Novak M, Kabat J, Wischik CM (1993) Molecular characterization of the minimal protease resistant tau unit of the Alzheimer's disease paired helical filament. EMBO J 12(1):365-370
47. Peeraer E, Bottelbergs A, Van Kolen K, Stancu IC, Vasconcelos B, Mahieu M, Duytschaever H, Ver Donck L, Torremans A, Sluydts E, Van Acker N, Kemp JA, Mercken M, Brunden KR, Trojanowski JQ, Dewachter I, Lee VM, Moechars D (2015) Intracerebral injection of preformed synthetic tau fibrils initiates widespread tauopathy and neuronal loss in the brains of tau transgenic mice. Neurobiol Dis 73:83-95

48. Planel E, Tatebayashi Y, Miyasaka T, Liu L, Wang L, Herman M, Yu WH, Luchsinger JA, Wadzinski B, Duff KE, Takashima A (2007) Insulin dysfunction induces in vivo tau hyperphosphorylation through distinct mechanisms. J Neurosci 27(50):13635-13648

49. Quinn JP, Corbett NJ, Kellett KAB, Hooper NM (2018) Tau Proteolysis in the Pathogenesis of Tauopathies: Neurotoxic Fragments and Novel Biomarkers. J Alzheimers Dis 63(1):13-33

50. Sanders DW, Kaufman SK, DeVos SL, Sharma AM, Mirbaha H, Li A, Barker SJ, Foley AC, Thorpe JR, Serpell LC, Miller TM, Grinberg LT, Seeley WW, Diamond MI (2014) Distinct tau prion strains propagate in cells and mice and define different tauopathies. Neuron 82(6):1271-1288

51. Scholl M, Lockhart SN, Schonhaut DR, O'Neil JP, Janabi M, Ossenkoppele R, Baker SL, Vogel JW, Faria J, Schwimmer HD, Rabinovici GD, Jagust WJ (2016) PET Imaging of Tau Deposition in the Aging Human Brain. Neuron 89(5):971-982

52. Schwarz AJ, Yu P, Miller BB, Shcherbinin S, Dickson J, Navitsky M, Joshi AD, Devous MD Sr, Mintun MS (2016) Regional profiles of the candidate tau PET ligand 18F-AV-1451 recapitulate key features of Braak histopathological stages. Brain 139(Pt 5):1539-1550

53. Takeda S, Wegmann S, Cho H, DeVos SL, Commins C, Roe AD, Nicholls SB, Carlson GA, Pitstick R, Nobuhara CK, Costantino I, Frosch MP, Muller DJ, Irimia D, Hyman BT (2015) Neuronal uptake and propagation of a rare phosphorylated high-molecular-weight tau derived from Alzheimer's disease brain. Nat Commun 6:84-90

54. Wang Y, Garg S, Mandelkow EM, Mandelkow E (2010) Proteolytic processing of tau. Biochem Soc Trans 38(4):955-961

55. Wischik CM, Novak M, Edwards PC, Klug A, Tichelaar W, Crowther RA (1988) Structural characterization of the core of the paired helical filament of Alzheimer disease. Proc Natl Acad Sci U S A 85(13):4884-4888

56. Wischik CM, Novak M, Thogersen HC, Edwards PC, Runswick MJ, Jakes R, Walker JE, Milstein C, Roth M, Klug A (1988) Isolation of a fragment of tau derived from the core of the paired helical filament of Alzheimer disease. Proc Natl Acad Sci U S A 85(12):4506-4510

57. Yanamandra K, Kfoury $N$, Jiang H, Mahan TE, Ma S, Maloney SE, Wozniak DF, Diamond MI, Holtzman DM (2013) Anti-tau antibodies that block tau aggregate seeding in vitro markedly decrease pathology and improve cognition in vivo. Neuron 80(2):402-414

58. Zilka N, Filipcik P, Koson P, Fialova L, Skrabana R, Zilkova M, Rolkova G, Kontsekova E, Novak M (2006) Truncated tau from sporadic Alzheimer's disease suffices to drive neurofibrillary degeneration in vivo. FEBS Lett 580(15):3582-3588

\section{Publisher's Note}

Springer Nature remains neutral with regard to jurisdictional claims in published maps and institutional affiliations. 